23 Correspondence to: emartens@umich.edu

$24{ }^{\#}$ Lead contact

25 Arbor, MI 48109 University, West Lafayette, IN 47907

Running Title: Bacteroides ribose utilization

\title{
A genetically adaptable strategy for ribose scavenging in a human gut symbiont plays a
}

diet-dependent role in colon colonization

Robert W. P. Glowacki ${ }^{1}$, Nicholas A. Pudlo ${ }^{1}$, Yunus Tuncil ${ }^{2,3}$, Ana S. Luis ${ }^{1}$, Anton I. Terekhov ${ }^{2}$, Bruce R. Hamaker ${ }^{2}$ and Eric C. Martens ${ }^{1, \#}$

${ }^{1}$ Department of Microbiology and Immunology, University of Michigan Medical School, Ann

${ }^{2}$ Department of Food Science and Whistler Center for Carbohydrate Research, Purdue

${ }^{3}$ Current location: Department of Food Engineering, Ordu University, Ordu, Turkey 


\section{Summary}

Efficient nutrient acquisition in the competitive human gut is essential for microbial

41 persistence. While polysaccharides have been well-studied nutrients for the gut microbiome,

42 other resources such as co-factors and nucleic acids have been less examined. We describe a

43 series of ribose utilization systems (RUSs) that are broadly represented in Bacteroidetes and

44 appear to have diversified to allow access to ribose from a variety of substrates. One Bacteroides

45 thetaiotaomicron RUS variant is critical for competitive gut colonization in a diet-specific

46 fashion. Using molecular genetics, we probed the nature of the ribose source underlying this diet-

47 specific phenotype, revealing that hydrolytic functions in RUS (e.g., to cleave ribonucleosides)

48 are present but dispensable. Instead, ribokinases that are activated in vivo and participate in

49 cellular ribose-phosphate metabolism are essential. Our results underscore the extensive

50 mechanisms that gut symbionts have evolved to access nutrients and how metabolic context

51 determines the impact of these functions in vivo.

52

53 
Symbiotic microorganisms that inhabit the human intestine complement digestive

57 capacity in numerous ways, with the most mechanistically understood examples involving

58 degradation and fermentation of chemically diverse fiber polysaccharides (Flint et al., 2012;

59 Porter and Martens, 2017). Host digestive enzymes of salivary, gastric, and pancreatic origin

60 target the major classes of dietary nutrients, notably fat, protein, and cooked or non-resistant

61 starches (Goodman, 2010; Iqbal and Hussain, 2009). In contrast, dietary fibers are degraded far

62 less, if at all, by host enzymes and instead require members of the gut microbiota for

63 transformation into host-absorbable short chain fatty acids (Macfarlane and Macfarlane, 2003).

64 As a consequence, dietary carbohydrates play an important role in shaping the composition and

65 physiology of the gut microbiota (David et al., 2014; Sonnenburg et al., 2016; Sonnenburg et al.,

66 2010). Unlike the aforementioned nutrients, the digestive fates of nucleic acids (from diet, host

67 or microbial origin), their component covalently linked ribo- and deoxyribonucleosides and

68 cofactors or modifications built from similar molecules are less understood. In particular, the

69 identities of common symbiotic human gut bacteria that are capable of utilizing these molecules

70 and the most relevant source(s) and forms of these substrates remain obscure. Since

71 enterohemorrhagic E. coli (EHEC) and other pathogenic E. coli have been shown to utilize

72 nutrients like ribose or deoxyribose or associated nucleic acids/nucleosides (Fabich et al., 2008;

73 Martinez-Jehanne et al., 2009), these substrates may represent unexplored nutrient niches that are

74 competed for by commensal and pathogenic microorganisms and therefore help mediate

75 colonization resistance against pathogens.

76 Some commensal and pathogenic human gut bacteria have known abilities to utilize free

77 ribose or deoxyribose, as well as (deoxy)ribonucleosides and nucleic acids. Specifically, strains

78 of mutualistic Lactobacillus (McLeod et al., 2011) and Bifidobacterium (Pokusaeva et al., 2010),

79 as well as pathogenic and non-pathogenic Escherichia coli (Fabich et al., 2008) and Salmonella

80 enterica (Harvey et al., 2011) have characterized mechanisms for ribose catabolism. Further, the

81 ability of EHEC to prioritize ribose as a nutrient in vivo is thought to provide an advantage over

82 commensal E. coli HS and may delineate different niches occupied by these strains (Maltby et

83 al., 2013). Additional systems containing nucleoside-cleaving enzymes have been defined in $E$.

84 coli and certain species of Corynebacterium isolated from feces (Hammer-Jespersen et al., 1971;

85 Kim et al., 2006). One of the more interesting groups of nutrients in this category that can be 
used by some gut bacteria is DNA. As demonstrated in E. coli, DNA serves as a sole-carbon

87 source through the action of competence genes and exonucleases (Finkel and Kolter, 2001;

88 Palchevskiy and Finkel, 2009). However, mechanisms for assimilating exogenous RNA have not

89 been explored.

90 Members of the phylum Bacteroidetes constitute a major portion of all bacteria found in

91 the human gut, and species within this phylum devote large proportions of their genomes

92 towards carbohydrate utilization via coordinately regulated polysaccharide utilization loci

93 (PULs). A number of PULs have been investigated in great depth in model species like

94 Bacteroides thetaiotaomicron and Bacteroides ovatus (Cuskin et al., 2015; Larsbrink et al., 2014;

95 Luis et al., 2018; Ndeh et al., 2017). Most of the characterized systems target dietary or host

96 polysaccharides, such as those found in plant cells, fermented foods or host mucosal

97 polysaccharides, while others have been definitively linked to degradation of less common

98 dietary substrates such as agarose and porphyran from edible seaweed (Hehemann, 2012;

99 Pluvinage et al., 2018). Despite variations in the substrates they target, the cellular "Sus-like

100 systems" encoded by Bacteroidetes PULs (Martens et al., 2009) are patterned in similar ways-

101 each containing one or more TonB-dependent receptors (SusC homologs) and adjacently

102 encoded substrate binding lipoproteins (SusD homologs). These two proteins form a complex

103 with extensive protein-protein interactions (Glenwright et al., 2017) and work with a variable

104 repertoire of cell surface and periplasmic carbohydrate-degrading enzymes, substrate binding

105 proteins and regulators to bind, degrade and import their specific substrates. However, despite

106 many studies examining the substrate specificity and function of Sus-like systems, many

107 additional PULs have been identified in the genomes of gut and environmental Bacteroidetes

108 without existing knowledge of their target substrates (Terrapon et al., 2018).

109 Here we describe a diverse set of gene clusters present in human gut and environmental

110 Bacteroidetes that are regulated by ribose, but have likely evolved to target a variety of different

111 ribose-containing nutrients. Using Bacteroides thetaiotaomicron (Bt) as a model, we investigated

112 a PUL of unknown function that is upregulated in vivo in multiple dietary conditions, including

113 in the absence of dietary fiber when this bacterium is forced to forage host-derived nutrients.

114 This gene cluster contains two predicted ribokinases and a nucleoside hydrolase (NH) among

115 other functions. Based on these predictions, we provide support for the hypothesis that this PUL

116 targets ribose as a nutrient, which may in many cases need to be cleaved from covalently linked 
117 sources, and show that it confers a diet-specific competitive advantage to Bt during in vivo

118 colonization. Surprisingly, the dietary condition during which this ribose utilization system (rus)

119 is required is a high-fiber diet that supplies a variety of other carbohydrate nutrients. $B t$ does not

120 require its two rus-encoded hydrolytic enzymes, or SusC/D-based transport for this diet-specific

121 competition. Instead, ribokinase function is essential, suggesting that sensing and metabolism of

122 simpler ribose and ribose-phosphate derivatives provides the key metabolic advantage in this

123 particular dietary condition. Taken together, our results reveal that a variety of host-associated

124 and terrestrial bacteria, including numerous human gut symbionts have evolved mechanisms to

125 scavenge ribose from various sources. The common regulation of a family of highly diversified

126 PULs by a ubiquitous simple sugar that occurs in a variety of molecular contexts, such as nucleic

127 acids, co-factors, modifications (ADP- and poly-ADP-ribose) and bacterial capsules, suggests

128 that these systems have been adapted at the enzymatic level to release ribose from these varied

129 sources, allowing Bacteroidetes to expand their colonization of diverse nutrient niches.

\section{Results}

132 A ribose-inducible gene cluster is highly active in vivo and required for fitness in a diet133 dependent fashion

134 Members of the prominent human gut bacterial phylum Bacteroidetes typically encode

135 coordinated degradative functions within discrete gene clusters called polysaccharide utilization

136 loci (PULs), facilitating identification of functions that work together to access a particular

137 nutrient (Martens et al., 2009). Previous work using gnotobiotic mice in which $B$.

138 thetaiotaomicron $(B t)$ is the only colonizing bacterium identified a locus (BT2803-2809) for

139 which all individual genes are upregulated between 10- and 139-fold in vivo, including in mice

140 fed diets with high and low dietary fiber (Fig. 1A). Under low fiber conditions, Bt's physiology

141 shifts to expression of genes involved in host glycan foraging (Bjursell et al., 2006; Martens et

142 al., 2008; Sonnenburg et al., 2005). Thus, the corresponding expression of BT2803-09 in the

143 absence of dietary fiber suggests that it also targets endogenous nutrients. Interestingly, the genes

144 in this locus were also most highly expressed in neonatal mice still consuming mothers milk,

145 which is not only fiber-deficient but also contains milk-derived ribose and nucleosides

146 (Schlimme et al., 2000). Typically, PULs involved in host glycan foraging encode enzymes 
147 required for liberating nutrients from mucus glycoproteins: fucosidase, sulfatase, $\beta$-galactosidase 148 and $\beta$-N-acetylhexosaminidase (Bjursell et al., 2006; Sonnenburg et al., 2005). However, some 149 predicted enzymes encoded in the BT2803-09 PUL (nucleoside hydrolase and ribokinases)

150 suggest a role in harvesting ribose from substrate(s) such as nucleosides or RNA, which could be 151 of host or gut bacterial origin. Previous studies have determined that $B t$ can grow on ribose as a 152 sole carbon source (Martens et al., 2011). However, the genes involved, the relevant source(s) of 153 this sugar, and whether it involves enzymatic liberation from complex sources remain unknown.

154 The architecture of the PUL spanning BT2803-09 revealed several unique features 155 compared to other PULs activated in low fiber diets (Fig. 1B). The immediate upstream gene 156 (BT2802) is predicted to have DNA-binding motifs and may act as a regulator but shares no 157 homology to regulators previously associated with PULs, the next two genes are predicted 158 ribokinases (BT2803 and BT2804), followed by genes encoding homologs of the Bacteroides 159 SusC and SusD outer-membrane proteins (BT2805, BT2806), a glycoside hydrolase of 160 unassigned family and function (BT2807), a predicted nucleoside hydrolase (BT2808), and a 161 sugar permease (BT2809). The enzymes encoded in this PUL suggested the hypothesis that it is 162 responsible for $B t$ 's ability to catabolize ribose and possibly liberate it from more complex 163 sources, such as RNA, nucleosides, or cofactors. To test if this gene cluster is transcriptionally 164 responsive to growth on ribose, we performed in vitro growth experiments in minimal-medium 165 (MM) containing ribose as the sole carbon source and measured expression of the genes BT2803166 09. All genes were activated 142-240 fold during growth on ribose compared to growth on a 167 MM-glucose reference (Fig. 1C). We next examined the contribution of this PUL to ribose 168 catabolism by deleting the entire PUL and upstream gene (BT2802). Consistent with an essential 169 role in ribose catabolism, loss of the PUL eliminated the ability to grow on free ribose (Fig. 1D). 170 Based on these findings, we classified this PUL as the $B t$ ribose utilization system, rus, with gene 171 annotations rusR (putative regulator), rusK1 and rusK2 (ribokinases 1 and 2), rusC (SusC-like),

$172 r u s D$ (SusD-like), rusGH (glycoside hydrolase), rusNH (nucleoside hydrolase), and rusT

173 (transporter), for genes BT2802-09 respectively. These in vitro results, combined with the

174 observation that rus exhibits high activity in the gnotobiotic mouse gut, led us to hypothesize that 175 the ability to utilize endogenous sources of ribose-containing nutrients is advantageous in vivo 176 during fiber-deficient diets. 
To test the above hypothesis, we inoculated 6-8 week old, germfree (GF) female Swiss-

178 Webster mice with a mixture of wild-type and $\Delta r u s B t$ strains $\left(\sim 10^{8}\right.$ total cfu/mouse, equal

179 amounts of each) and maintained mice on either a fiber-rich (FR) diet containing several

180 unprocessed plant-derived fiber polysaccharides or a fermentable fiber-free (FF) diet consisting

181 mainly of glucose, protein, lipids, and cellulose (Desai et al., 2016). We measured the relative

182 abundance (by qPCR) of each strain for 42 days in DNA extracted from feces. Surprisingly, and

183 in contrast to our initial hypothesis, the $\Delta r u s$ strain was strongly outcompeted ( 100 -fold) only in

184 mice fed the FR diet (Fig. 2A). In contrast, in mice fed the FF diet, $\Delta r u s$ exhibited similar

185 abundance to wild-type throughout the experiment (Fig. 2B). A similar competitive defect of the

$186 \Delta$ rus strain in mice fed the FR diet was observed in separate experiments with 12-week-old

187 female mice and 6-8 week old male mice (Fig. S1A,B), suggesting the competition is not

188 influenced by sex or age within the range tested. The defect associated with the FR diet was not

189 due to lack of colonization or persistence in vivo, as the levels of each strain were similar over

190 time in mice colonized with either strain alone (Fig. S1C,D). Additionally, the defect seen in the

191 FR diet could not be attributed to the wild-type strain exhibiting different expression of the rus

192 PUL, as wild-type $B t$ exhibited similarly high levels of rus expression in mice fed either diet

193 when present alone or in competition with the $\Delta r u s$ mutant (Fig. S1E). GC-MS analysis of the

194 two diets revealed that ribose was present only in the FR diet, in levels similar to other common

195 monosaccharides, and in an acid-hydrolyzable (i.e., covalently linked) form, but not detectably

196 as a free sugar. This suggested the presence of a ribose-containing molecule(s), such as RNA,

197 nucleosides or cofactors, which may be scavenged by $B t$ in mice fed this diet (Fig. S1F).

198 However, in cecal contents of mice monoassociated with wild-type or $\Delta r u s$ strains and fed the

199 FR diet, ribose was undetectable while other sugars present in acid-hydrolyzed extracts of the

200 uneaten FR diet—and likely deriving from fibers such as arabinan and arabinoxylan — could still

201 be measured (Fig. S2, note that we determined through control experiments that the limit of

202 detection of ribose in the complex milieu of cecal contents is near the amount observed in the FR

203 diet, although the levels of other sugars seems to have been concentrated as much as 2-fold

204 during digestion). This unexpected result led us to conclude that although ribose is present in the

205 FR diet it may be depleted or absorbed during transit through the upper GI, such that it is not

206 detectable even in the cecal contents of mice colonized by $\Delta r u s$, which cannot use this sugar.

207 Nevertheless, to directly test if dietary ribose from different sources can impact $B t$ in the distal 
gut, we colonized three separate groups of GF mice with a mixture of wild-type and $\Delta r u s$ strains

209 and maintained them on the FF diet that does not elicit a competitive defect for $\Delta r u s$. After 14

210 days of stable competition between strains, water was supplemented with either $1 \%$ ribose, $1 \%$

211 RNA, or $1 \%$ nucleosides $(0.25 \% \mathrm{w} / \mathrm{v}$ each of uridine, thymidine, 5-methyl uridine, and cytidine).

212 The results clearly show that free ribose present in the drinking water elicits a competitive fitness

213 defect for the $\Delta r u s$ strain similar in magnitude and with slightly faster timing, to the defect in

214 mice fed the FR diet (Fig. 2C). In contrast, little defect, if any, was observed in mice switched to

215 water containing the nucleoside mix or RNA (Fig. 2D,E). Similar to mice fed just FR or FF,

216 there was similar expression of the rus locus in all of the supplemented water conditions,

217 suggesting that levels of rus expression did not account for the various fitness outcomes (Fig.

218 2F). While our findings above imply that little free ribose is present in the FR diet and even the

219 acid-hydrolyzed, covalently-linked form(s) may be removed before reaching the distal gut, our

220 results with dietary additions of ribose-containing nutrients clearly indicate that free dietary

221 ribose, but not RNA or nucleosides, is a form of this nutrient capable of driving abundance

222 changes in Bt populations.

223 A subset of ribose-utilization functions is required for competitive colonization in mice

224 The experiments described so far utilized a mutant lacking all 8 rus genes, but only a

225 subset of the functions may be important for competition with wild-type in mice fed the FR diet.

226 Because biochemical approaches failed to reveal a clear ribose source that drives the competitive

227 advantage associated with rus expression in the FR diet, we took a molecular genetic approach to

228 probe the required enzymatic and transport functions. We constructed single and double gene

229 deletions based on predicted functionality (Fig. 1B), performing additional competitive

230 colonization experiments in FR diet-fed mice. Each individual mouse group was inoculated with

231 wild-type $B t$ and one of the following competing strains ( $\Delta r u s K 1 / 2, \Delta r u s C / D, \Delta r u s G H / N H$,

$232 \Delta r u s T$, or $\Delta r u s R$ ). Surprisingly, the only strain that exhibited a competitive fitness defect similar

233 to the full $\Delta r u s$ mutant was $\Delta r u s K 1 / K 2$, which lacks both predicted ribokinases (Fig. 3A). In

234 contrast, the other four deletion strains exhibited equal or better competition compared to wild-

235 type (Fig. 3B-E). Notably, the $\Delta r u s G H / N H$ strain, which lacks $r u s$-associated hydrolase

236 functions, exhibited a significant competitive advantage ( $\sim 100$-fold better than wild-type). These

237 results clearly suggest that the required functions underlying the competitive defect in the $\Delta r u s$ 
238 strain are encoded by the rusK1 or rusK2 genes, while expression of the other genes provide no 239 advantage in this context, and perhaps even a fitness disadvantage in the FR diet. To further

240 address which of the ribokinases is important in vivo we repeated the above competition with

241 single $\triangle r u s K 1$ and $\triangle r u s K 2$ deletion strains. Each of these single kinase mutants also competed

242 better than wild-type, suggesting that they are redundant and need to be lost together to elicit a

243 defect (Fig. 3F,G). As in previous experiments, we could not attribute variations in competitive

244 behavior to a significant difference in rus expression in wild-type $B t$, which was elevated in all

245 cases (Fig. 3H).

\section{A subset of Rus functions is required for sensing and utilization of ribose containing}

247 nutrients in vitro

248 The results described above clearly indicate a diet-specific advantage for $B t$ strains that

249 possess the rus-encoded ribokinases. To further define this system's function, we tested our

250 panel of deletion mutants in a variety of growth conditions, including free ribose, nucleosides,

251 RNA, and other sources of this sugar. Consistent with our in vivo data, a mutant lacking both

$252 r u s K 1$ and rusK2 could not grow on free ribose (Fig. 4A). However, arguing against purely

253 redundant functions as concluded above, the mutant lacking just rusK2 displayed a complete loss

254 of growth phenotype, while a mutant lacking only rusK1 reproducibly displayed a substantial

255 growth lag, but eventually grew with slightly slower rate compared than wild-type (Fig. 4B,C).

256 The delayed growth phenotype of this mutant might be attributed to genetic suppressor mutations

257 or another heritable alteration, since cells that eventually grew were able to grow quickly on

258 ribose after being isolated and passaged in rich media (Fig. S3A). Deletion of the flanking gene

$259 \operatorname{rus} R$, a candidate transcriptional regulator, was also unable to grow on ribose, suggesting that,

260 although it is not transcriptionally activated in response to ribose, its product plays an essential

261 role in ribose catabolism (Fig. 4D). The $\Delta r u s T$ strain exhibited an increased lag, slower growth

262 rate and lower overall growth level compared to wild-type (Fig. 4E). Unlike the $\Delta r u s K 1$ mutant

263 this mutant did not exhibit increased growth after passaging and re-testing, suggesting that

264 suppressor mutations are not involved and rather another, lower-affinity pentose sugar permease

265 is present to import ribose. Lastly, the $\triangle r u s C / D$ strain consistently exhibited a longer growth lag

266 on ribose, suggesting that although ribose should freely diffuse across the outer membrane, the

267 RusC/D complex on the cell surface might increase affinity for this sugar leading to accelerated 
268 growth (Fig. 4F, reference growth of wild-type $B t$ on ribose is shown here). All of the other 269 single or double deletion mutants tested ( $\Delta r u s C, \Delta r u s D, \Delta r u s G H, \Delta r u s N H, \Delta r u s G H / N H$ ), 270 exhibited no measurable difference in growth parameters compared to wild-type $B t$ when grown 271 on ribose (Fig. S3B-F, Table S1).

272 Since they are larger and more complex, we hypothesized that utilization of molecules in 273 which ribose is covalently linked to other ligands would require additional rus-encoded 274 functions. To test this, we assayed growth of our rus mutants and wild-type $B t$ on nucleosides 275 and RNA. Wild-type $B t$ displayed poor growth, when observed at all, on all nucleosides tested 276 (uridine, cytidine, 5-methyl uridine, thymidine, inosine, xanthosine, adenosine) as well as on 277 RNA (Fig. S3G,H, Table S1). We hypothesized that free ribose may need to be present to 278 activate transcription of the rus locus, generating proteins necessary for catabolism of these 279 substrates. To test this, we determined a concentration $(0.5 \mathrm{mg} / \mathrm{ml})$ at which ribose elicited 280 strong rus expression but little if any measurable growth (Fig. S3I,J). We then re-evaluated the 281 ability of wild-type $B t$ to grow on nucleosides in the presence of this low ribose concentration, 282 observing considerably higher levels of total growth on pyrimidine nucleosides (Fig. 4G). While 283 growth was still comparably poor relative to growth on pure ribose, increased growth was not 284 observed when we doubled the nucleoside concentrations, suggesting that something else about 285 growth on nucleosides limits growth (Fig. S3K). Growth on nucleosides was eliminated in 286 mutants lacking the full locus (Fig. 4H), either or both ribokinases (rusK1, rusK2, and $287 r u s K 1 / K 2$ ), the candidate regulator (rusR) and the putative transporter (rusT). Each of these 288 phenotypes was similar to those observed for growth on free ribose, except the $\Delta r u s K 1$ and $289 \Delta$ rusT mutants, which eventually grew with reduced rate on ribose, but not on nucleosides (Fig. 290 S3L-P). Growth on RNA alone was not observed, even after addition of ribose, suggesting that $291 B t$ does not produce sufficient extracellular RNAse and phosphatase enzymes required to liberate 292 nucleosides from this polymer. Therefore, we tested if exogenous RNase A and intestinal 293 alkaline phosphatase (IAP), which are present in the gut from host pancreatic secretions (RNAse) 294 or native to the enterocyte brush boarder and secreted in luminal vesicles (IAP), could enhance 295 growth on RNA at physiologically relevant concentrations (McConnell et al., 2009; Weickmann 296 et al., 1984). When these host-derived enzymes were supplemented in media, growth on RNA 297 was appreciably greater than in their absence (Fig. 4I), which was not attributable to $B t$ growing 298 on the exogenous enzymes alone (Fig. S3Q). As with individual nucleosides, reductions or 
299 eliminations in growth on enzyme-degraded RNA were observed in mutants lacking the entire 300 rus locus, rusK1, rusK2, rusK1/K2, rus $T$, and $\operatorname{rusR}$ (Fig. 4J). In addition to free nucleosides and 301 those derived from RNA, we also determined that $B t$ is able to utilize the pentoses deoxyribose 302 and lyxose, as well as ADP-ribose and UDP-galactose: each of these required the presence of

303 both a low amount of ribose and the rus locus. Sixteen additional substrates did not support $B t$ 304 growth under any conditions (Table S1).

305 Inconsistent with our initial hypothesis, mutants lacking functional rusC, rusD, rus $G H$,

$306 \mathrm{rusNH}$, rus $C / D$ or $r u s G H / N H$ encoded products, exhibited total growth levels comparable to

307 wild-type on both nucleosides and degraded RNA (Fig. 4I, S3R-W). These results suggested that 308 other genes in $B t$ encode functions responsible for cleavage and utilization of free nucleosides 309 and those liberated from RNA. To identify other functions involved in utilization of nucleosides, 310 we searched the $B t$ genome for functions from known nucleoside scavenging systems (NSSs), 311 identifying several candidates. We made deletions of 4 genes predicted to encode nucleoside 312 cleavage and import functions, BT0184, BT1881, BT4330, and BT4554, which are predicted to 313 encode a uridine kinase, a purine nucleoside phosphorylase, a nucleoside permease, and a 314 pyrimidine nucleoside phosphorylase, respectively. We tested growth of these strains on 315 nucleosides (Fig. 5A-D) and only one strain ( $\triangle B T 4554)$ displayed loss of growth on all 316 nucleosides tested, suggesting that it encodes an essential enzyme for cleaving nucleosides and 317 works upstream of also required rus functions. The $\triangle B T 4330$ mutant also exhibited reductions in 318 growth on uridine, cytidine, and 5-methyl uridine, while only slight defects were seen in 319 thymidine (Fig. 5A-D). Further, the $\triangle B T 0184$ mutant displayed enhanced growth that began 320 quicker than wild type and reached a higher total growth level on all nucleosides, except the 321 deoxyribonucleoside thymidine. This phenotype could be due to its role in 5' phosphorylating 322 scavenged nucleosides and shunting them towards anabolic pathways, such that when it is 323 deleted catabolic growth is enhanced. Lastly, $\triangle B T 1881$ did not display any detectable growth 324 defects compared to wild-type, suggesting that the product of this gene is not essential for 325 pyrimidine nucleoside catabolism. Interestingly, although growth on nucleosides in some NSS 326 mutants were reduced or eliminated compared to wild-type, this phenotype did not extend to 327 growth on RNA, as the mutant strains exhibited similar levels of growth as wild-type (Fig. 328 S4A,B). These results suggest that, while Rus functions are required to use RNA-derived 329 nucleosides, the NSS functions interrogated here are not essential for catabolism of RNA-derived 
330 nucleosides, which might vary in their nucleoside ratios or oligomer length and could be

331 assimilated via additional pathways.

\section{Rus enzymes are active towards ribose-containing substrates or nucleosides}

The results described above suggest that the product of the rusNH gene, if functional, is superfluous to pyrimidine nucleoside salvage, since deletion of BT4554 eliminated growth on these nutrients. To test if this enzyme is actually functional, we produced a recombinant $B t$

337 RusNH by over-expression in E. coli and performed substrate cleavage assays. We first used $p$ 338 nitrophenyl- $\beta$-D-ribofuranoside (pNP-ribose), on which this enzyme was active and determined

339 the $\mathrm{pH}$ optimum to be 6.7. We next tested the cleavage specificities and affinities of ribo- and

340 deoxyribonucleosides in a UV-based assay (Liang et al., 2008), observing that RusNH has broad,

341 but relatively weak activity compared to other nucleoside hydrolases towards all nucleosides

342 tested (Table S2). This broad range of catalytic activities suggests that this enzyme belongs to

343 the inosine-uridine-preferring family of nucleoside hydrolases (IUNH) as predicted by

344 annotation. However, despite containing the canonical N-terminal DXDXXXDD motif involved

345 in binding of ribose and coordination of $\mathrm{Ca}^{2+}$ ions (Fig. S4C), the kinetic values of RusNH are

346 not within the range of previously characterized IUNH hydrolases from other organisms (Table

347 S2) (Parkin et al., 1991; Shi et al., 1999; Versées and Steyaert, 2003). Although we attempted to

348 directly measure enzyme affinities by $\mathrm{km}$ determination, the activity was too weak to reach

349 Vmax at the concentrations tested, further suggesting that RusNH is not primarily responsible for 350 nucleoside cleavage.

351 Because RusNH has relatively weak activity towards nucleosides, we hypothesized that

352 the predicted glycoside hydrolase, RusGH, could have activity on nucleosides since it has not

353 been assigned a previously defined glycoside hydrolase (GH) family. A potentially important

354 role for RusGH was further suggested by its possible location on the cell surface, which was

355 suggested by a signal peptidase II secretion-lipidation signal and confirmed by antibody staining,

356 whereas RusNH lacks this signal and appears to be secreted into the periplasm (Fig. S4D, data

357 not shown for RusNH, which was not similarly detected on the cell surface despite being

358 detectable in whole cell lysates by western blot). We therefore produced recombinant RusGH

359 and tested a broad-range of pNP-based substrates in several buffer conditions and found

360 optimum conditions to be pNP-ribose at $\mathrm{pH} 9.0$ (Table S2). Arguing against a major role in 
361 cleavage of any of the substrates tested, RusGH displayed only weak activity on pNP-ribose that

362 was too slow for accurate kinetic determinations and no detectable activity on other pNP

363 substrates after 24 hours. Interestingly, the weak activity displayed was calcium or divalent

364 cation dependent as addition of EDTA completely eliminated activity (Table S2). When RusGH

365 was tested for the ability to cleave nucleosides for $24 \mathrm{~h}$, no liberation of ribose was observed.

366 Additional testing on a panel of glycans that are capable of supporting $B t$ growth failed to reveal

367 any additional activity. Thus, although the $B t$ Rus harbors two enzymes with demonstrable but

368 weak activities, roles for these enzymes remains enigmatic, although it is possible that larger

369 polymers exist that are the targets for these enzymes.

370

\section{Dynamics of rus transcript activation and global responses to ribose catabolism}

Our in vivo and in vitro data support an important role for some Rus functions in

373 utilization of ribose and ribonucleosides, although a critical part of the latter pathway hinges on

374 the function of an unlinked gene, BT4554. Because Rus function and a small amount of free

375 ribose is essential for utilization of nucleosides via the BT4554 phosphorylase, we sought to

376 determine the requirements for activating expression of rus genes as well as the presence of other

377 global responses that ribose may induce. We hypothesized that the critical Rus functions for

378 responding to free ribose are the reaction products from one or both kinases, RusK1, RusK2, and

379 also require the putative regulator RusR, and the permease, RusT. To test this, we examined the

380 kinetics of rus transcriptional responses when $B t$ was exposed to ribose. We grew our wild-type

381 and mutant strains in medium containing glucose as a sole carbon source, washed them in a

382 carbohydrate-free medium, transferred the bacteria into medium containing ribose as the only

383 carbon source and monitored rus transcript accumulation over time (Fig. 5E). Our results show

384 that wild-type $B t$ achieves close to maximum activation between 15-30 minutes post-exposure,

385 with continued slow increase in expression. Interestingly, the $\Delta r u s K 2$ strain, which cannot grow

386 on ribose, still generated near wild-type levels of transcript on the same time scale. In contrast,

387 the $\Delta r u s K 1$ mutant, which exhibits an extensive lag before growth on ribose, was unable to

388 quickly generate transcript on a $2 \mathrm{hr}$ time scale (Fig. 5E), but eventually achieves near wild-type

389 rus expression once actively growing on ribose (Fig. S4E). This latter result is consistent with

390 our previous observation with the Bt starch utilization system (Cameron et al., 2014) that growth

391 phenotypes characterized by protracted lag periods are in some cases due to deficient ability to 
392 sense an activating sugar cue. As expected based on the single kinase deletions, the $\Delta r u s K 1 / K 2$

393 double mutant was unable to generate transcript, while the $\Delta r u s T$ exhibited only slightly lower

394 expression than wild-type (Fig. 5E). The $\Delta r u s R$ mutant only achieved partial ( $\sim 10 \%)$ activation

395 in response to ribose, which supports the idea that RusR is required for positive transcription

396 activation and the partial expression could be due to the absence of glucose repression during

397 ribose exposure. Finally, we measured rus expression dynamics in our $\Delta r u s C$ and $\Delta r u s D$ strains,

398 with the hypothesis that these outer membrane proteins may increase the cell's affinity for ribose

399 leading to more rapid activation, but failed to detect any differences in expression compared to

400 wild type. (Fig. S4F). Further, to rule out nucleosides (processed to ribose-1-P via BT4554)

401 serving as a possible inducing molecule we monitored rus transcript over time when wild type $B t$

402 was exposed to either uridine or inosine in the absence of ribose and did not see rus activation

403 (Fig. S4G).

404 The two ribose-inducible kinases encoded in the Bt rus locus are predicted to generate

405 products that are part of the pentose phosphate pathway (PPP), with known ribokinases adding

406 phosphate to the 5' position, although we cannot rule out generation of (1-, 5- or 1-/5-phosphate).

407 Thus, we hypothesized that growth on exogenous ribose may affect expression of a more global

408 regulon that could contribute to the in vivo competitive defect associated with the FR diet. We

409 initially probed expression of genes involved in metabolizing the other pentoses, xylose and

410 arabinose, revealing that growth on ribose leads to increased expression of genes involved in

411 arabinose utilization, but only to $\sim 10-20 \%$ of levels when grown directly in arabinose (Fig. 5F),

412 however the same effect was not seen for genes involved in xylose metabolism (Fig. S4H). To

413 extend these results, we performed RNAseq-based whole-genome transcriptional profiling on

414 wild-type $B t$ grown on ribose or glucose to address if growth on ribose elicits a broader

415 metabolic response. The data indeed reveal a global response in which 81 genes are differentially

416 expressed according to the parameters used. Unexpectedly, many of the genes (46\%) belong to

417 other PULs or metabolic pathways, with most of the remaining genes encoding hypothetical

418 functions or undefined pathways. Notable changes included upregulation of a previously defined

419 PUL for fructose and $\beta 2,6$-linked fructan metabolism (BT1757-1765; average upregulation of

420 15-fold), which interestingly liberates fructose that initiates the PPP (Sonnenburg et al., 2010).

421 At the same time, two other PULs of unknown specificity were repressed (BT3024-3027,

422 BT3344-3347). Further, Bt has a partial TCA cycle pathway (Pan and Imlay, 2001) of which 
423 several genes leading to generation of succinate and propionate were upregulated, while genes

424 predicted to participate in sugar-phosphate isomerization and metabolism, are strongly repressed

425 (BT2156-2159; average of 24-fold) (Fig. 5G). An experiment to test the hypothesis that cross-

426 regulation between ribose metabolism and the fructan PUL is the cause of the FR-specific in vivo

427 competition defect did not support this model since a strain lacking rus in the context of an

428 inability to use fructans was still outcompeted by a strain lacking only fructan use, and this was

429 not due to changes in rus activation (Fig. S4I-J). Thus, we conclude that the critical role of the

430 Rus ribokinases for $B t$ fitness in vivo is contingent upon a more complex set of metabolic

431 interactions that require the generation of a phosphorylated ribose signal(s) that better equips $B t$

432 to compete in the guts of mice fed the FR diet.

An enzyme-diversified family of Rus systems exists throughout the Bacteroidetes

While the Bt rus encodes two enzymes with relatively weak activity and little contribution to the in vivo phenotype observed on the FR diet, the architecture of this system

437 suggests that it is equipped to liberate ribose from sources more complex than ribonucleosides.

438 This led us to hypothesize that rus-like systems are found in other gut isolates and perhaps more

439 broadly across the Bacteroidetes phylum. To test this, we measured the growth ability of 354

440 different human and animal gut Bacteroidetes belonging to 29 species in MM-ribose, revealing

441 that ribose utilization is widely but variably present in indvidual species and strains (Fig. 6A,

442 Fig. S5A). To determine if sequenced representatives of the species/strains that grow on ribose

443 contain a homolog of the experimentally validated Bt rus, we conducted a comparative genomics

444 analysis by searching for homologs of Bt rus within these gut isolates. This comparison revealed

445 that all of the sequenced strains with the ability to grow on ribose also possessed a candidate rus-

446 like PUL, while none of the strains unable to grow on ribose had a homologous gene cluster.

447 Interestingly, our comparative genomics analysis revealed very similar homologs of some

448 rus genes in sequenced gut isolates (e.g., Prevotella) that were not tested in our initial survey.

449 When we expanded the search to include these species, plus Bacteroidetes isolates found in other

450 body sites and in the environment, we detected rus-like systems across the Bacteroidetes

451 phylum, with the systems found in the genus Bacteroides being most similar to the prototype

452 from Bt. Remarkably, we identified a total of 70 different rus configurations, ranging from

453 simple two gene units (permease and kinase, which do not formally define a PUL), to rus PULs 
454 containing as many as 36 genes (Fig. 6B, Fig. S5B). This analysis revealed that for almost all 455 rus-like systems, the following genes are present: $r u s C$ and $r u s D$ homologs, an upstream rusR

456 homolog or to a lesser extent, different regulator types (LacI is most prevalent after RusR), either 457 one or two ribokinase genes, and a rusT homolog. Perhaps most intriguingly, the predicted 458 enzymes found in different rus-like systems are exceptionally variable. There are at least 22 459 different predicted glycoside hydrolase families, along with ADP-ribosylglycohydrolases (ADP$460 \mathrm{RGH}$ ), carbohydrate esterases, nucleoside hydrolases, and other predicted enzymatic activities.

461 This plethora of enzymatic potential encoded in rus homologs across the Bacteroidetes phylum 462 suggests individual species or strains target different ribose-containing nutrients. To further 463 connect these predicted rus-like systems with ribose utilization, we probed the transcriptional 464 response of 8 different systems during growth on MM-ribose, finding that all strains tested 465 exhibited 100-1000 fold upregulation relative to a MM-glucose reference (Fig. 6C).

\section{Discussion}

467 Diet impacts the human gut microbiota in many ways and members of the prominent 468 Bacteroidetes phylum have developed diverse strategies to liberate sugars from often very 469 complex dietary fiber polysaccharides (Luis et al., 2018; Ndeh et al., 2017). Such abilities equip 470 these bacteria to compete for and utilize dietary and endogenous nutrients to sustain their 471 populations. Diet, microbiome- and host-derived RNA, nucleosides, cofactors and other sources

472 of ribose have been largely unexplored as potential nutrients scavenged by members of the gut

473 microbiota. Our findings not only demonstrate that $B t$ utilizes free- and covalently-linked sources

474 of ribose, but that this metabolic capability contributes to competitive fitness in vivo in a diet-

475 dependent fashion-likely through a more complicated metabolic mechanism(s) than just

476 acquisition of ribose. It is also clear from our comparative genomics investigation that the ability 477 to access ribose, probably from diverse sources, extends across the Bacteroidetes phylum and is 478 present in many strains from the human gut, oral cavity, and environment.

479 Based on our results we have developed a working model of ribose utilization built 480 around findings from the Bt rus PUL and canonical metabolic pathways (Fig. 7). Components 481 encoded directly within this PUL are required for import, recognition and phosphorylation of 482 ribose and presumably more complicated molecules that have yet to be discovered, with RusK1 483 being important for generating the signal for rus induction (Fig. 5E). The identity of this 
484 inducing molecule is also unknown, but is unlikely to be ribose-5-P, which is presumably made

485 during non-inducing growth on glucose via the PPP to synthesize nucleic acid components and

486 histidine. Data also suggest it may not be ribose-1-P, which is the product of nucleoside

487 phosphorylase and exposure to nucleosides did not activate expression (Fig. S4G); although, we

488 cannot rule out that in the absence of a low amount of ribose, nucleosides are not transported to

489 be cleaved by BT4554. This leaves ribose-1,5-diphosphate as a potentially unique candidate. Our

490 model also postulates that the ribokinases are required for catabolism of nucleosides, which are

491 primarily imported and cleaved by the product of the BT4554 phosphorylase. This

492 interconnection may stem from the predicted lack of a dedicated phosphopentomutase in $B t$,

493 which would be required to convert ribose-1-P generated by BT4554 into ribose-5-P required for

494 entry into the catabolic branch of the pentose phosphate pathway (PPP). As such, at least one

495 additional kinase would be required to create ribose-1,5-PP, a precursor of PRPP that provides an

496 alternative path back to the catabolic branch of PPP, although an orthologous enzyme that

497 catalyzes this transition has also not been identified in Bt. In light of a recent study

498 demonstrating that the Bt nucleoside phosphorylase (BT4554) has the ability to modify the levels

499 of a nucleoside-containing drug (Zimmermann et al., 2019), our findings hold important

500 implications for how native responses to this overlooked nutrient source can affect the fitness of

501 bacteria and subsequent interactions with the metabolome around them. They also extend the

502 range of enzymatic functions that are encoded in Bacteroidetes PULs beyond the known

503 examples of carbohydrate modifying enzymes (Cuskin et al., 2015; Porter and Martens, 2017)

504 and proteases (Nakjang et al., 2012; Renzi et al., 2015) to include those acting on nucleosides.

505 Similar to only one other PUL that was previously characterized in $B t$, the prototypic rus

506 PUL is activated in response to a monosaccharide (Fig. 1C), rather than the more common

507 sensory strategies driven by oligosaccharide cues. The previously described system for fructan

508 utilization is activated by free fructose, which occurs in two common linkages $(\beta 2,1$ and/or $\beta 2,6)$

509 in polysaccharides for which various Bacteroides strains have acquired substrate-specific

510 enzymes in their respective fructan utilization systems (Sonnenburg et al., 2010). Comparable to

511 the ribose utilization system described here, the fructan system also contains a dedicated

512 permease and a kinase revealing that these two systems are similarly patterned around a core

513 monosaccharide utilization pathway. Taken together, these observations may indicate that one

514 mechanism for PUL evolution and diversification is building enzyme systems, which liberate the 
515 same sugar from multiple sources, around a central hub of sugar utilization functions such as

516 import and phosphorylation. While covalently-linked fructose is present in only a few known

517 forms (inulin, levan, graminins) from plant fructans and microbial capsules (van Arkel et al.,

518 2013), ribose is much more widely present but frequently exists in a form linked to a variety of

519 other non-sugar moieties.

520 Often, the enzymatic content of Bacteroidetes PULs provides a window into the fine

521 linkage structure of the nutrient(s) that any given system has evolved to target for degradation.

522 For example, investigation of $B t$ PULs required for degrading $\alpha$-mannan polysaccharides derived

523 from fungal cell walls revealed enzymes specific for unique modifications in individual fungal

524 species (Saccharomyces cerevisiae, Schizosaccharomyces pombe and Candida albicans) (Cuskin

525 et al., 2015; Temple et al., 2017). An additional study of xyloglucan utilization PULs across

526 multiple species revealed variants that contain $\alpha$-fucosidases, presumably equipping them to

527 target fucosylated xyloglucans present in the cell walls of lettuce and other leafy greens

528 (Larsbrink et al., 2014). Ribose is present in many diverse sources with different linkages,

529 including RNA and nucleosides, bacterial capsules, cofactors such as NAD, cellular modification

530 like (poly) ADP-ribose and more exotic molecules such as microcins (Duquesne et al., 2007;

531 Knirel et al., 2002). By coupling ribose monosaccharide sensing, import, and phosphorylation to

532 an adaptable series of enzymes encoding a large repertoire of cleavage functions, we hypothesize

533 that the different ribose utilization systems across the Bacteroidetes are tuned to liberate ribose

534 from a wide variety of sources. The breadth of this enzymatic diversity found across the phylum

535 is emphasized by the presence of at least 22 different glycoside hydrolase families, nucleoside

536 cleaving enzymes (nucleoside hydrolases, ADP-ribosylglycohydrolases, NAD-hydrolases),

537 esterases, and more totaling a minimum of 70 different configurations of ribose utilization

538 systems. Interestingly, many prominent Bacteroides species isolated from the human gut have

539 ribose utilization systems with similar enzymatic content, suggesting that there may be a

540 narrower range of common nutrient(s) targeted by these organisms in their colonic habitat.

541 However, two of the most complex systems observed also exist in human gut Bacteroidetes

542 (Alistipes senegalensis and Dysgonomonas mossi, Fig. 6B), suggesting that they have also

543 adapted to more complex molecules. Finally, the large number of rus-encoded glycoside

544 hydrolases suggests that a prominent target of these systems may be ribose-containing

545 polysaccharides used to construct the capsules and exopolysaccharides of other gut bacteria, a 
546 dimension of gut microbial ecology and glycobiology that has been poorly explored (Porter and

547 Martens, 2017) despite strains of some common species like $E$. coli using ribose to construct 548 capsules (Hackland et al., 1991).

549 Since homologs of rus are present in numerous members of the human gut microbiota,

550 we hypothesized that at least one source of ribose scavenged in vivo would be endogenous RNA

551 from bacteria themselves or turnover of host cells. Consistent with this idea, previous studies

552 demonstrated that Bt rus is expressed to high levels in vivo during dietary conditions in which

553 fiber is depleted or during $B t$ only chemostat cultures in which biofilms develop and cellular

554 debris including RNA may accumulate (TerAvest, 2013). If an endogenous source of ribose is

555 mostly what is scavenged in vivo, competition during low fiber diet feeding should have revealed

556 a bigger defect for the $\Delta r u s$ strain compared to wild-type. However, absence of rus did not result

557 in a competitive defect unless ribose was provided in the context of FF diet as a free

558 monosaccharide. Similar supplementation with nucleosides or RNA did not reveal the same

559 effect, suggesting that despite $B t$ being able to utilize nucleosides and enzyme-degraded RNA as

560 carbon sources in vitro, dietary sources of these molecules might be absorbed before reaching the 561 distal gut.

562 Despite lack of identification of a nutrient source(s) that drives the FR-specific diet

563 effect, our study clearly indicates the importance of rus in vivo, especially in the FR diet, but also

564 when ribose is present in addition to a low fiber diet. What is more surprising about this result is

565 that monosaccharide analysis of the diets revealed ribose as only a minor constituent of the fiber

566 rich diet in a covalently linked form. One possible mechanism for why the $\Delta r u s$ strain is severely

567 outcompeted in this diet is that utilization pathways for other nutrients may be interconnected

568 with ribose sensing in $B t$ and that small amounts of ribose below the limit of detection, but

569 sufficient to upregulate rus, act to enhance utilization of other nutrients in vivo or optimize co-

570 utilization of multiple nutrients. This idea is supported by our RNA-seq analysis, specifically the

571 ribose-induced upregulation of genes involved in fructan and arabinose metabolism. Further,

572 several genes responsible for functions encoded in the TCA cycle are upregulated in ribose,

573 suggesting that ribose may remodel the metabolic landscape in such a way to promote faster

574 assimilation of nutrients. Therefore, the ability to sense and respond to ribose via rus provides

575 the observed competitive advantage despite only low concentrations of ribose are present from

576 the diet. This phenomenon suggests that nutrients such as monosaccharides should not be 
577 overlooked for their intrinsic simplicity and assumption that they do not affect the gut 578 microbiota, as they may still impart changes to individual species' global metabolism.

579 A poignant example of how competitive survival in the gut requires evolution of complex

580 nutrient acquisition strategies is exemplified by the $B t$ rhamnogalacturonan-II (RG-II) acquisition

581 system. RG-II consists of 13 different sugars connected through 21 unique linkages and $B t$

582 contains three co-expressed PULs to scavenge this nutrient, using all of the individual products

583 but one. The results described here highlight how the survival of related bacteria from the human

584 gut and other ecosystems has driven adaptations to sense and scavenge ribose, a ubiquitous sugar

585 that occurs in a number of different molecules, which has apparently led to enormous species and

586 strain level variation in the enzymes present in rus loci. This evolution is analogous to a

587 molecular "Swiss-army knife", in which the core function is utilization of the simple sugar ribose

588 but the various blades and other implements represent the enzymes that equip a given system to

589 sense, import or harvest ribose from one or more sources. This molecular adaptability is

590 particularly important in the context of the nutrient niche hypothesis of gut bacterial survival.

591 While some nutrients may be scarce compared to common and abundant dietary fiber

592 polysaccharides, competition for these lower abundance nutrients may be less intense and

593 organisms capable of accessing them could thereby occupy a stable niche. While a number of gut

594 bacteria, including pathogens, are capable of utilizing free ribose, the Bacteroides may have

595 developed a more sophisticated ability to scavenge multiple sources of ribose from covalently

596 linked forms. From this perspective, understanding the struggle to access this "simple" nutrient

597 may reveal additional layers underpinning the interplay between native gut mutualists and

598 invading pathogens.

600 Materials and methods

601

602 Bacterial strains, culturing conditions, and molecular genetics.

603 B. thetaiotaomicron ATCC 29148 (VPI-5482) and its genetic variants, as well as other

604 Bacteroides strains used in this study, were routinely grown in tryptone-yeast extract-glucose

605 (TYG) broth medium (Holdeman, 1977), in minimal medium (MM), plus a defined carbon

606 source (Martens et al., 2008), or on brain heart infusion agar with 10\% defibrinated horse blood

607 (Colorado Serum Co.). Unless otherwise noted, carbon sources used in MM were added to a final 
608 concentration of $5 \mathrm{mg} / \mathrm{ml}$. Cultures were grown at $37^{\circ} \mathrm{C}$ in an anaerobic chamber $\left(10 \% \mathrm{H}_{2}, 5 \%\right.$

$609 \mathrm{CO}_{2}$, and $85 \% \mathrm{~N}_{2}$; Coy Manufacturing, Grass Lake, MI). Genetic deletions and mutations were 610 performed by counter-selectable allelic exchange as previously described (Koropatkin et al., 611 2008). Primers used in this study are listed in Table $\mathbf{S 6}$ To quantify growth on carbon sources 612 and examine mutant phenotypes, increase in culture absorbance $(600 \mathrm{~nm})$ in $200 \mu l$ cultures in 613 96-well plates was measured at 10 minute intervals for at least 96 hours on an automated plate

614 reader as previously described (Martens et al., 2011). To achieve consistent and robust growth on 615 nucleosides and other covalently linked sources of ribose, free ribose was added at a final 616 concentration of $0.5 \mathrm{mg} / \mathrm{ml}$ to $\mathrm{MM}$ containing $5 \mathrm{mg} / \mathrm{ml}$ of carbon source. Growth on $5 \mathrm{mg} / \mathrm{ml}$ of 617 MM containing Type IV Torula yeast RNA (Sigma) was obtained by adding 100 units of calf618 intestinal alkaline phosphatase (CIP) (New England Biolabs) and 2mg/ml RNase A (Sigma).

619 Growth parameters and conditions for all substrates are summarized in Table S1.

\section{Genetic manipulation and recombinant protein purification in $\boldsymbol{E}$. coli}

622 To create a nucleoside hydrolase-free expression background, E. coli BL21-AI ${ }^{\mathrm{TM}}$ One

623 Shot $^{\circledR}$ cells (Invitrogen) were manipulated using lambda red recombineering to introduce genetic 624 deletions of the ribose-inducible hydrolase genes ( $\mathrm{rih}$ ) to avoid contaminating activity in 625 downstream applications of purified proteins (Petersen and Moller, 2001). The E. coli gene 626 deletion procedure developed by Datsenko and Wanner (Datsenko and Wanner, 2000) was 627 followed with few modifications. Briefly, BL21-AI cells were transformed with the pKD46 628 plasmid. Transformed cells were grown overnight in $\mathrm{LB}+\mathrm{Amp}^{100}$ and sub-cultured, when the 629 culture absorbance $(600 \mathrm{~nm})$ reached 0.1 , L-arabinose was added to $10 \mathrm{mM}$ final concentration 630 to induce the $\mathrm{P}_{\mathrm{BAD}}$ promoter of pKD46, cells were allowed to grow to an OD between 0.6-0.8 631 and made competent for electroporation by cold water washes and stored in $10 \%$ glycerol 632 aliquots. For recombineering, 400ng of gel-purified PCR product was added to freshly made 633 cells and incubated for 10 minutes on ice, electroporated in a $2 \mathrm{~mm}$ gap cuvette at $2500 \mathrm{~V}$, 634 recovered in $1 \mathrm{ml} \mathrm{LB}$ at $30^{\circ} \mathrm{C}$ for 5 hours. All knockouts were made sequentially in this manner 635 via introduction of the following antibiotic cassettes (spectinomycin from K11497 for $\Delta$ rihA; 636 hygromycin from $\mathrm{K} 11521$ for $\Delta r i h B$; gentamicin from $\mathrm{K} 11590$ for $\Delta r i h C$ ), and the following 637 concentrations of antibiotic were used for selection: $\operatorname{Spec}^{80}$, $\operatorname{Hygro}^{200}, \mathrm{Gent}^{10}$. Following 638 construction of the last deletion, the pKD46 plasmid was heat-cured by passaging twice at $42^{\circ} \mathrm{C}$ 
639 in LB. To better control background expression of the $\mathrm{T} 7$ promoter, the $\mathrm{T} 7$ lysozyme containing 640 plasmid, pLysS from BL21 (DE3) (Lucigen) was introduced into the strain via $\mathrm{Ca}^{2+}$ chemical

641 competence/heat shock. Protein purification was accomplished using the pETite N-His vector 642 (Lucigen). PCR primers were designed to amplify products for BT2807 and BT2808 containing 643 all amino acids downstream of the predicted signal peptide sequences, residues 22-539 for

644 BT2807 and residues 22-338 for BT2808, amplified and transformed into Hi-Control 10G cells 645 according to manufactures protocol (Lucigen, Expresso ${ }^{\mathrm{TM}} \mathrm{T} 7$ cloning and expression system).

646 pETite plasmids containing BT2807 or BT2808 were transformed into $E$. coli strains TUNER or 647 BL21-AI $\triangle r i h A B C+$ pLysS, respectively. A single colony was grown in $5 \mathrm{~mL}$ of LB+Kan ${ }^{50}$ for 648 16h. This pre-inoculum was added to to $1 \mathrm{~L}$ of Terrific-Broth with 50ng/ul of Kanamycin and 10 $649 \mathrm{ng} / \mathrm{ul}$ of Chloramphenicol (BT2808) or 50ng/ul of Kanamycin (BT2807) and culture was grown 650 with shaking at $37^{\circ} \mathrm{C}$ until absorbance 0.4 at $600 \mathrm{~nm}$. BT2807 and BT2808 cells were induced 651 with a final concentration of $0.2 \mathrm{mM}$ or $1 \mathrm{mM}$ IPTG and $0.2 \% 20 \mathrm{mM}$ L-arabinose, respectively, 652 and temperature was reduced to $16^{\circ} \mathrm{C}$ and outgrown overnight. The recombinant proteins were 653 purified by immobilized metal ion affinity chromatography using cobalt (BT2807) or nickel654 affinity (BT2808) columns was accomplished as described previously (Cameron et al., 2014). 655

\section{Measurements of transcriptional responses by qPCR}

$657 \quad B t$ and other Bacteroides strains were grown to mid-exponential phase 0.6-0.8 658 (absorbance at 600nm) in MM+ribose, MM+arabinose, MM+xylose, or MM+glucose, two 659 volumes of RNA protect added, followed by centrifugation and storage of cell pellets at $-80^{\circ} \mathrm{C}$.

660 Total RNA was extracted using the RNeasy mini kit buffers (Qiagen) and purified on RNA661 binding spin columns (Epoch), treated with TURBO DNaseI (Ambion) or DNase I (NEB) after 662 elution and purified again using a second RNeasy mini kit isolation column. Reverse 663 transcription was performed using SuperScript III reverse transcriptase and random primers 664 (Invitrogen). The abundance of each target transcript in the resulting cDNA was quantified using 665 either KAPA SYBR® FAST qPCR mix (KAPA Biosystems) or a homemade qPCR mix as 666 described here: each $20 \mathrm{uL}$ reaction contained 1X Thermopol Reaction Buffer (NEB), 125uM 667 dNTPs, 2.5mM MgSO4, 1 X SYBR Green I (Lonza), 500nM gene specific or 65nM 16S rRNA 668 primer and 0.5 units Hot Start Taq Polymerase (NEB), and 10ng of template cDNA. For the 669 KAPA mix, $400 \mathrm{nM}$ of primers specific for genes in the rus locus of $B t$ or the rusC-like gene of 
670 other Bacteroides species or $62.5 \mathrm{nM}$ of $16 \mathrm{~S}$ rRNA primers and 10ng of template cDNA as 671 described previously (Pudlo et al., 2015). Using the ddCT method, raw values were normalized

672 to $16 \mathrm{~S}$ rRNA values and then MM+ribose values were referenced to the values obtained in

$673 \mathrm{MM}+$ glucose to obtain a fold-change. Measurements of transcriptional response over time in

$674 \mathrm{MM}+$ ribose or nucleosides was performed similarly to previously described (Cameron et al.,

675 2014). Briefly, strains were grown in TYG, subcultured 1:50 into MM+glucose, at mid-

676 exponential phase, cells were washed twice in MM-no carbon and resuspended in MM+ribose

677 with time points being taken every $5 \mathrm{~min}$ for the first $30 \mathrm{~min}$ and every $15 \mathrm{~min}$ for a total of 120

$678 \mathrm{~min}$. Measurements of transcriptional responses to varying amounts of ribose were performed

679 similarly as above, but only one time point was taken after $30 \mathrm{~min}$ of exposure to varying

680 concentration of MM+ribose ranging from $0.0005 \mathrm{mg} / \mathrm{ml}$ to $5 \mathrm{mg} / \mathrm{ml}$.

681

682 Gnotobiotic mouse experiments

683 All experiments involving animals, including euthanasia via carbon dioxide asphyxiation, 684 were approved by the University Committee on Use and Care of Animals at the University of 685 Michigan (NIH Office of Laboratory Animal Welfare number A3114-01) and overseen by a 686 veterinarian. Six to eight-week-old, germfree female Swiss-Webster mice were initially 687 maintained on the standard, fiber-rich lab diet (LabDiet 5010, LabDiet, St. Louis, MO), where 688 appropriate, mice were switched to a fiber-free diet (Envigo-Teklad TD 130343) and maintained 689 for one week prior to colonization with $B t$ strains. After stable colonization had been observed, at 690 day 14 some groups of mice were provided water ab libitum containing one of the following: $1 \%$ 691 ribose, $1 \%$ Nucleoside mixture ( $0.25 \%$ thymidine, $0.25 \%$ uridine, $0.25 \% 5$-methyl uridine, and $6920.25 \%$ cytidine) or Type VI torula yeast RNA. DNA was extracted from fecal pellets throughout 693 the experiment and strain abundance was quantified as described previously (Desai et al., 2016).

694 Relative abundance of each strain was normalized to the original abundance on day of gavage 695 (day 0). Post-sacrifice, cecal contents were collected, flash frozen and stored at $-80^{\circ} \mathrm{C}$. RNA was 696 extracted as described previously (Porter and Martens, 2017), briefly, RNA was phenol-

697 chloroform treated and ethanol precipitated, DNA removed by treatment with TURBO ${ }^{\mathrm{TM}}$ DNaseI 698 (Ambion), followed by purification using RNeasy mini kit (Qiagen) according to manufactures 699 instructions. 
Purified recombinant BT2807 and BT2808 proteins were used as antigens to raise rabbit 703 polyclonal antibodies (Cocalico Biologicals, Inc, Stevens PA). Antibody specificity and cellular

704 localization for BT2807 and BT2808 were determined by western blotting of wild-type and

705 relevant mutant strains and by immunofluorescent microscopy of $B t$ VPI-5482 grown in

$706 \mathrm{MM}+$ glucose or MM+ribose. Growth conditions are described above, cells for WB were grown

707 to mid-log optical absorbance $(600 \mathrm{~nm})$ 0.6-0.7 or 0.4-0.5 for IF. Western blots of $B t$ whole cell

708 lysates were performed using the primary, polyclonal antibodies mentioned above and secondary

709 antibody conjugated to goat anti-Rabbit IgG conjugated alkaline phosphatase (Sigma) and

710 detected with NBT/BCIP (Roche). Surface expression of BT2807 or BT2808 was examined by

711 staining with a BT2807- or BT2808-specific primary antibody in non-permeabilized

712 formaldehyde-fixed $B t$ cells and detected with Alexa-Flour ${ }^{\circledR} 488$ conjugated goat anti-Rabbit

713 IgG secondary (Molecular Probes), as described previously (Ref). Cells were imaged on an IX-

71470 inverted microscope (Olympus) with images captured at 100x magnification. A minimum of

715 five fields of view per slide was observed with $\mathrm{n}=2$ biological replicates.

\section{Comparative genomics of rus PULs across Bacteroidetes genomes}

718 A total of 354 different Bacteroidetes strains were tested for growth on ribose as a sole

719 carbon source as shown in Fig. 6A and summarized in Table $\mathbf{S 4}$ The ability to use ribose is

720 shown in the context of a previously published human gut Bacteroidetes phylogeny that used 14

721 conserved genes across phylum members (Larsbrink et al., 2014). To search for rus locus

722 homologs across the Bacteroidetes phylum, we used the amino acid sequences of the rusK1,

723 rusK2, rus $T$, and rusR genes from the $B t$ type strain as deletion of these genes yielded growth

724 defects on ribose. We searched the Integrated Microbial Genomes (IMG) database (current as of

725 May 2018) and performed phylum-level BLAST searches with an E-value cutoff of 1e-50. We

726 chose this stringent cutoff as initial searches using lower values obtained many non-specific hits

727 of genes encoding other kinases and permeases that did not appear to be specific for ribose,

728 including in the Bt VPI-5482 genome for which Rusk1 and RusK1 are the only kinases able to

729 promote ribose growth. After we completed our search for rusK, rusT, and rusR homologs we

730 used the Gene Neighborhood tool in IMG to determine if these hits were located directly next to

731 other genes involved in ribose utilization. The presence of a minimum of two adjacent rus gene 
732 homologs was required to count the presence of a candidate utilization locus. Following this first

733 round of searching we observed that many of the rus loci contained one or more nucleoside

734 cleaving enzymes such as homologs of Bt rusNH or ADP-ribosylglycohydrolases (RGH) and

735 upstream putative regulatory genes. To give our search more power and potentially find

736 additional rus homologs we performed additional searches with the same E-value threshold for

737 homologs of Bt rusNH, or homologs of the ADP-RGH in B. xylanisolvens XB1A. When

738 assembling the comparative genomics data, gene names and glycoside hydrolase family

739 assignments are shown as predicted in IMG or by BLAST of the amino acid sequence of

740 individual genes. Further, in refinement, a handful of genes were found below our E-value, but

741 included in the table as it is clear from gene neighborhood views in IMG that it is likely to be

742 part of a rus locus. Types of rus have been assigned based only on gene content and arrangement

743 as a way to indicate differences, however subtle. In completing our table we have included the

744 bit score as well as the amino acid \% identities compared to Bt rus genes or Bx XB1A ADP-RGH

745 genes. All of the positive gene hits with locus tag information, isolation location, and other

746 relevant strain information is summarized in Table S5.

\section{RNAseq analysis}

To determine the global transcriptional response to growth in ribose as the sole carbon

750 source, $B t$ cells were grown overnight in rich TYG media then transferred to fresh MM

751 containing either $5 \mathrm{mg} / \mathrm{ml}$ glucose or $5 \mathrm{mg} / \mathrm{ml}$ ribose. Cells were then grown until mid-log phase

752 (absorbance between 0.6-0.8) and two volumes of RNA Protect (Qiagen) were added to cells.

753 RNA was isolated as described above and purified whole RNA was then rRNA depleted using

754 the Ribo-Zero Bacterial rRNA Removal Kit (Illumina Inc.) and concentrated with the RNA

755 Clean and Concentrator-5 kit (Zymo Research Corp, Irvine, CA). Samples were multiplexed for

756 sequencing on the Illumina HiSeq platform at the University of Michigan Sequencing Core. Data

757 was analyzed using Arraystar software (DNASTAR, Inc.) using RPKM normalization with

758 default parameters. Gene expression in ribose was compared to gene expression in a glucose

759 reference. Genes with significant up- or down-regulation were determined by the following

760 criteria: genes with and average fold-change $\geq 5$-fold and with at least $2 / 3$ biological replicates

761 with a normalized expression level $\geq 1 \%$ of the overall average RPKM expression level in either

762 glucose or ribose, and a p-value $<0.05$ ( $\mathrm{t}$ test with Benjamini-Hochberg correction) (Table S3). 


\section{Enzyme assays}

Recombinant proteins purified in E. coli, were used to determine enzyme kinetics for RusGH and RusNH. For RusNH we used a $p$-nitrophenol-ribofuranoside substrate with absorbance readings at $405 \mathrm{~nm}$ over a 24 -hour period as described previously (Desai et al., 2016), with modifications for using purified protein instead of crude extract, using $0.5 \mathrm{mM}$ of enzyme in a buffer containing $20 \mathrm{mM}$ Hepes and $100 \mathrm{mM} \mathrm{NaCl}$, at $\mathrm{pH} 6.7$ at $37^{\circ} \mathrm{C}$ and continuous absorbance readings. For RusGH, a panel of other 4-nitrophenol based substrates in addition to $p$-NP-ribofuranoside were tested at $\mathrm{pH} 9.0$ in $100 \mathrm{mM}$ Tris at $37^{\circ} \mathrm{C}$ for $16 \mathrm{~h}$ with $1.5-15 \mu \mathrm{M}$ of enzyme and using endpoint absorbance measurements. Ion requirements of the RusGH were assayed in $p$-NP-ribofuranoside by addition of divalent cations in the form of $\mathrm{CaCl}_{2}, \mathrm{ZnCl}_{2}$, or

$773 \mathrm{MgCl}_{2}$, at 2, 5, or $10 \mathrm{mM}$ concentrations, or in the presence of $10 \mathrm{mM}$ EDTA. Specificity and

774 kinetic parameters for RusNH on natural nucleoside substrates were determined as described 775 previously using a UV-based assay (Parkin et al., 1991). Briefly, a 96-well, UV-compatible 776 microplate (Santa Cruz Biotechnologies) was used with substrate concentrations ranging from $0.025 \mathrm{mM}-2.5 \mathrm{mM}$, and enzyme concentrations of $0.25-1 \mathrm{uM}$. Assays were immediately read after addition of enzyme by continuous reading of absorbance at $262 \mathrm{~nm}$ or $280 \mathrm{~nm}$ with time points taken every 2.5 minutes over $12-24$ hours at $37^{\circ} \mathrm{C}$. Volume was $250 \mathrm{uL}$ in all assays and carried out in buffer containing $20 \mathrm{mM}$ Hepes and $100 \mathrm{mM} \mathrm{NaCl}$, at $\mathrm{pH} 6.7$, adjusted with acetic acid. As a measure of catalytic efficiency, $\left(K_{\text {cat }} / K_{M}\right)$ was unable to be determined by classical MichaelsMenton kinetics as Vmax was never reached and therefore $\mathrm{Km}$ values were not accurate, so we used a previously established method of estimating this value (Ndeh et al., 2017). Briefly, we used a single substrate concentration to calculate $\left(k_{\text {cat }} / K_{\mathrm{M}}\right)$ and checked to be $<<K_{\mathrm{M}}$ by halving and doubling the substrate concentration and observing a proportionate increase or decrease in 786 rate. Therefore the equation, $V_{0}=\left(k_{\mathrm{cat}} / K_{\mathrm{M}}\right)$ [S][E] was used to calculate $k_{\mathrm{cat}} / K_{\mathrm{M}}$ in our case. For,

787 RusGH a panel of other 4-nitrophenol based substrates in addition to $p$-NP-ribofuranoside were

788 tested at $\mathrm{pH} 9.0$ in $100 \mathrm{mM}$ Tris at $37^{\circ} \mathrm{C}$ for $16 \mathrm{~h}$ with $1.5-15 \mu \mathrm{M}$ of enzyme with endpoint

789 absorbance measurements. Ion requirements of the RusGH were assayed in $p$-NP-ribofuranoside

790 by addition of divalent cations in the form of $\mathrm{CaCl}_{2}, \mathrm{ZnCl}_{2}$, or $\mathrm{MgCl}_{2}$, at 2, 5, or $10 \mathrm{mM}$

791 concentrations, or in the presence of $1 \mathrm{mM}$ EDTA. The RusGH was tested against a panel of 792 oligosaccharides, nucleosides and nucleotides. Briefly, the reactions were performed with $10 \mu \mathrm{M}$ 793 of RusGH, $8 \mathrm{mg} / \mathrm{ml}$ substrate or $5 \mathrm{mM}$ monosaccharide in $50 \mathrm{mM}$ TRIS pH 9.0 at $37^{\circ} \mathrm{C}$ for $16 \mathrm{~h}$. 
794 A control reaction was performed in the same conditions without enzyme. The activity was

795 qualitative determined by thin layer chromatography. $6 \mu 1$ of the reaction was spotted on foil

796 backed silica plate (Silicagel 60, 20 x 20, Merck) and develop in butanol:acetic acid:water 2:1:1

797 (mobile phase). The products of the reaction were detected by immersing the TLC plate in

798 developer (sulphuric acid/ethanol/water 3:70:20 v/v, orcinol $1 \%$ ) for 30 seconds and heating to

$799100{ }^{\circ} \mathrm{C}$ for 2 minutes. A standard of ribose was run in all TLC plates.

801 Determination of free and acid hydrolysable monosaccharide content in diets and cecal 802 contents using GC/MS

803 Prior to analysis, diets were ground to a fine powder using a blender followed by mortar

804 and pestle, while cecal contents were dried by lyophilization. Samples were analyzed for free and

805 linked monosaccharides using the following method described in (Pettolino et al., 2012). In brief,

806 all reactions began with 1-3mg of sample and samples were hydrolyzed in 100ul of 2.5 M TFA

807 for $90 \mathrm{~min}$ at $121^{\circ} \mathrm{C}$. Samples were allowed to cool to room temperature (RT) and myo-inositol

808 was added as an internal standard (20ul of $2.5 \mathrm{mg} / \mathrm{ml})$ and dried under nitrogen. 150ul of

809 methanol was added, dried and repeated once more. Dried samples were then reduced by

810 dissolving in $50 \mathrm{ul}$ of $2 \mathrm{M} \mathrm{NH}_{4} \mathrm{OH}$ followed by addition of $50 \mathrm{ul}$ of freshly made $1 \mathrm{M} \mathrm{NaDB}_{4}$ in

$8112 \mathrm{M} \mathrm{NaOH}$. This mixture was sonicated in a water bath for $1 \mathrm{~min}$, followed by incubation at room

812 temperature for 2.5 hours. 23ul of glacial acetic acid was added and samples dried and

813 evaporated $2 \mathrm{x}$ with $250 \mathrm{ul}$ of $5 \%(\mathrm{v} / \mathrm{v})$ acetic acid in methanol, followed by $2 \mathrm{x}$ evaporation with

$814250 \mathrm{ul}$ of methanol, drying after each step. Acetylation was done by addition of $250 \mathrm{ul}$ acetic

815 anhydrate and sonicated $5 \mathrm{~min}$ followed by incubation at $100{ }^{\circ} \mathrm{C}$ for 2.5 hours. $2 \mathrm{ml}$ of $\mathrm{ddH}_{2} \mathrm{O}$

816 was added and sample vortexed to dissolve residue, followed by room temperature incubation for

$81710 \mathrm{~min} .1 \mathrm{ml}$ of dichloromethane (DCM) was added and vortexed followed by centrifugation at

$8182000 \mathrm{rpm}$ for $2.5 \mathrm{~min}$. The aqueous phase was discarded and the DCM phase washed $2 \mathrm{x}$ with 2

$819 \mathrm{ml}$ of $\mathrm{ddH}_{2} \mathrm{O}$. DCM phase was dried and residue dissolved in $250 \mathrm{ul}$ acetone. For free

820 monosaccharide analysis the initial hydrolysis step with TFA was not performed. To establish a

821 limit of detection in cecal contents, varying amounts of ribose $(0.00002-0.2 \mathrm{mg}$, in 10-fold

822 increments) were added at the same time as the myo-inositol standard to establish percent

823 recovery throughout the methods used. Acetylated samples were analyzed on a gas

824 chromatography (Agilent Technologies model 7890A) coupled mass spectrometer (Agilent 
825 Technologies model 5975C) using a fused silica capillary column $(60 \mathrm{~m} \times 0.25 \mathrm{~mm} \times 0.2 \mu \mathrm{m}$ SP826 2330, Supelco Analytical).

827

828

829

830 Acknowledgements

831

832

833

R.W.P.G. was supported by the NIH Molecular Mechanisms of Microbial Pathogenesis Training Program (T32 AI007528) as well as a University of Michigan Rackham Merit Fellowship. We thank the support of the Germ Free Mouse Facility at the University of Michigan for assistance with in vivo experiments. This work was supported by National Institutes of Health grants R21AI-128120, R01-GM-099513, R01-DK-118024 (all to E.C.M). 
Figure 1. Bt upregulates a PUL for ribose metabolism in vivo and in vitro in response to

838 ribose. (A) In vivo gene chip data (Bjursell et al., 2006; Sonnenburg et al., 2005) displaying fold839 change relative to in vitro growth in minimal medium (MM), plus glucose for BT2803-09 in

840 mice fed high fiber (dark and light green bars) or low fiber diets, including pre-weaned, suckling

841 mice (red and purple bars, respectively). (B) Genomic architecture of the rus locus spanning

842 genes BT2802-09 with names and predicted functions. (C) In vitro transcriptional response of rus

843 genes in $B t$ grown on ribose as a sole carbon source, measuring fold-change relative to growth on

844 a MM+glucose reference ( $n=3$, error bars are SD of the mean). (D) Growth on minimal media

845 containing ribose $(5 \mathrm{mg} / \mathrm{ml})$ as the sole carbon source in wild-type $B t$ (black) or a strain lacking

$846 r u s$ (red) through 96 hours of incremental readings (minimum of $\mathrm{n}=5$ separate replicates).

Figure 2. The locus BT2802-09 confers a competitive advantage in vivo in a diet-dependent

849 context. For all panels, relative abundance is shown on a log scale for wild-type (black) and

$850 \Delta r u s, B T 2802-09$ (red) strains and as measured by qPCR from fecal samples of gnotobiotic mice

851 ( $n=4$ for each experiment) colonized with a mixture of the two strains indicated at day 0. (A)

852 Mice continuously fed a high fiber diet (green arrow) for the entire duration. (B) Mice

853 continuously fed a low fiber diet (pink arrow) for the entire duration. (C-E) Mice initially

854 maintained on the FF-diet for two weeks and then at day 14 given ad libitum access to water

855 containing $1 \% \mathrm{w} / \mathrm{v}$ ribose (C), 1\% w/v of RNA from type IV Torula yeast RNA (D), or a 1\% w/v

856 mixture of nucleosides $(0.25 \%$ each of uridine, cytidine, thymidine, and 5-methyl uridine). In

857 each panel the duration of the water supplementation is shaded in either blue, orange, or purple,

858 representing the different substrates. (F) rus transcript levels measured by qRT-PCR probing the

859 rusC gene from in vivo cecal contents, bar colors correspond to the background shading in panels

860 A-E. Error bars in all panels display the standard error of the mean for each time point.

862 Figure 3. Ribokinases rusK1/K2 are sufficient to confer a competitive advantage in vivo.

863 (A-G) In all experiments, 6-8 week old germfree Swiss-Webster mice were fed the fiber rich

864 (FR) diet and gavaged with a mixture of both wild-type Bt (black) and the mutant strain indicated

865 (red) in nearly equivalent levels. Relative abundance is displayed over a 42-day experiment 
866 identically to Figure 2. In all panels error bars represent standard error of the mean of four 867 biological replicates. (H) rus transcript levels measured by qRT-PCR probing the rusC gene 868 from in vivo cecal contents for bacterial populations in each panel.

Figure 4. The Bt rus PUL encodes functions required for growth on ribose containing

871 nutrients. (A-E) Growth curves of rus deletion strains on ribose (red) or glucose (black) that

872 exhibit defects for growth on ribose compared to wild-type $B t$ (see panel F. for a wild-type

873 reference curve for growth on ribose). (F) Growth of wild-type and the $\Delta r u s C / D$ strains are

874 shown for glucose and ribose, revealing slightly weaker growth for the $r u s C / D$ mutant. (G-H)

875 Growth of wild-type (G) or $\Delta$ rus $(\mathrm{H})$ on different nucleosides in the presence of $0.5 \mathrm{mg} / \mathrm{ml}$ ribose

876 (yellow line is media with only $0.5 \mathrm{mg} / \mathrm{ml}$ ribose). (I-J) Growth of wild-type and rus deletion

877 strains on $5 \mathrm{mg} / \mathrm{ml}$ yeast RNA and media containing RNase A and IAP. Strains displaying

878 similar growth profiles as wild-type are in (I), while strains showing a reduction in growth are

879 shown in $(\mathrm{J})$. Note that in panels G-H the $\mathrm{y}$-axis scale is reduced. Curves shown in each panel are

880 the average of a minimum of 8 technical replicates.

\section{Figure 5. Requirement of some nucleoside scavenging systems, dynamics of $B t$ rus} 883 activation and global responses to ribose

884 (A-D) Growth curves of predicted nucleoside scavenging mutants ( $\triangle B T 0184$, red; $\triangle B T 1881$, 885 green; $\triangle B T 4330$, purple; or $\triangle B T 4554$, orange) and wild type $B t$ (black) on uridine, cytidine, 5886 methyl uridine or thymidine. (E) Bt rusC transcriptional response as measured by qRT-PCR over 887 time after mid-log phase cells actively growing in MM-glucose $(5 \mathrm{mg} / \mathrm{ml})$ were transferred to 888 MM-ribose $(5 \mathrm{mg} / \mathrm{ml})$. Samples were taken every 5 minutes for the first 30 minutes and then 889 every 15 minutes after until 120 minutes. Strains are color coded according to the legend: wild 890 type (black), $\Delta r u s K 1$ (green), $\Delta r u s K 1 / K 2$ (orange), $\Delta r u s R$ (red), $\Delta r u s K 2$ (purple), and $\Delta r u s T$

891 (blue). Shown are the averages of $n=3$ separate experiments (different days), error bars indicate 892 the standard error of the mean. (F) Transcriptional responses of genes in the arabinose gene 893 cluster BT0348-0355 during growth on $5 \mathrm{mg} / \mathrm{ml}$ ribose (orange) or $5 \mathrm{mg} / \mathrm{ml}$ arabinose (purple), 894 compared to $B t$ grown in MM-glucose ( $\mathrm{n}=3$ bars and error bars represent mean plus standard 895 error). (G) Select RNAseq results indicating a global transcriptional response during growth on 896 MM-ribose compared to a MM-glucose reference by fold-change in gene expression in each 
897 condition. Matching bar colors indicate genes are in the same locus or PUL. Additional genes

898 listed in gray bars are not genomically linked to adjacent-coordinately regulated genes. Bars

899 represent the mean plus standard deviation of 3 replicate experiments.

900

901 Figure 6. Ribose utilization is present throughout the Bacteroidetes phylum and rus 902 homologs appear in many configurations with highly variable enzyme content.

903 (A) Phylogeny of 29 human gut Bacteroidetes species (plus a P. copri root) showing the

904 sampling depth of the 354 strains surveyed for growth on ribose and the penetrance of ribose

905 utilization within each species. Outer black circles at tree tips are sized to represent the number

906 of strains sampled within each species, the inner red circles are sized to indicate the number of

907 strains for which ribose $(5 \mathrm{mg} / \mathrm{ml}$ ) supports growth (each strain was tested at least 2 times for

908 growth). (B) Comparative genomics of several variants of homologs of the rus locus discovered

909 throughout the Bacteroidetes phylum as described in Methods. The schematic of gene

910 annotations shows the vast enzymatic potential contained in different rus loci from the species

911 and strains indicated. Same background fill color indicates the same predicted function(s), no-

912 fill, white backgrounds indicate hypothetical/unknown functions. Adjacent to each locus

913 schematic is the species in which each rus homolog is present followed by a parenthetical

914 number denoting the number of sequenced isolates containing the indicated type of rus

915 architecture. Each architecture is assigned an arbitrary type number denoting the different gene

916 content and/or organization. All genes are sized relative to actual length within and between

917 genomes. All of the species shown here are human or mammalian gut isolates, a broader

918 representation of rus diversity is shown in Fig. S6 and includes rus homologs from

919 environmental and oral Bacteroidetes. Abbreviations: (GH*, Glycoside hydrolase of unknown

920 family/function; BACON, Bacteroidetes-Associated Carbohydrate-binding Often N-terminal

921 domain; DHDPS, dihydrodipicolinate synthase; LacI, predicted lacI-type transcriptional

922 regulator; MFS, Major-facilitator superfamily of transporters; ADP-RGH, ADP-ribosyl

923 glycoside hydrolase; DNAH, DNA helicase; PBS, Polysaccharide Biosynthesis and export of O-

924 antigen and techoic acids; DPP7, Dipeptidyl-Peptidase 7 (serine peptidase); GT, Glycosyl

925 Transferase). For simplicity, gene labels are only shown once with the top-most gene given a

926 label (i.e., RusNH in green). However, due to similar colors, genes annotated as BACON, LacI,

927 MFS and aryl sulfatase are labeled throughout). Asterisks next to the organism name indicate 
928 that the rus homolog type was shown to be upregulated during growth on ribose as the sole

929 carbon source. (C) Fold-change of rus $C$-like transcript from the indicated species during growth

930 on $5 \mathrm{mg} / \mathrm{ml}$ of ribose as a sole carbon source compared to growth in MM-glucose. Error bars

931 show the SEM of $\mathrm{n}=3$ biological replicates.

933 Figure 7. Model of ribose utilization in $\boldsymbol{B t}$

934 A schematic of $B t$ metabolism of ribose and related molecules based on the data shown,

935 predicted KEGG metabolism maps of the pentose phosphate pathway, and gene annotations.

936 Ribose is depicted as a pink star, its official symbol nomenclature for glycans symbol, with

937 appended phosphate or bases shown as appropriate (phosphate, yellow; uridine, blue; 5-methyl

938 uridine, green; cytidine, dark pink; guanosine, red). Extracellular ribose should diffuse across the

939 outer membrane and, once in the periplasm, taken up by the high-affinity ribose permease

940 (RusT) or an alternative sugar permease. Transported ribose is then phosphorylated by RusK1

941 and RusK2 yielding unspecified ribose-phosphates, with the product of at least RusK1 being

942 required for rus transcriptional activation. Canonical pathways exist for assimilating ribose-

943 phosphates into either catabolic pathways or synthesis of nucleic acids and histidine, although

944 the precise entry points for RusK1/K2 derived metabolites is unknown, as are the

945 interconversions catalyzed by steps that are not predicted to be present in $B t$ (dashed pathways

946 with red "x" marks). Nucleosides require the additional presence of transport machinery (e.g.,

947 BT4330) and nucleoside phophorylase activity (e.g., BT4554) in order to enter the cell and be

948 catabolized in a rus-dependent fashion. Nucleoside transport across the outer membrane in $E$.

949 coli requires a Tsx-like porin, although highly similar candidates were not found in Bt.

951 Figure S1. The Arus and wild-type strain colonization, rus expression in all diets, and 952 dietary monosaccharide analysis of FR and FF diets.

953 Data is related to Figure 2. Additional in vivo competition or monocolonization experiments

954 performed in Swiss Webster mice fed the fiber rich (FR) diet. Relative abundance of wild type

955 (black) vs. $\Delta r u s$ strain (red) were measured in (A) 12 week old female mice or (B) 6-8 week old

956 male mice. (C) 6-8 week old female mice mono-associated with either wild-type $B t$ (pink circles)

957 or $\Delta$ rus strain (green circles) and absolute abundance was assayed by dilution plating from fresh

958 fecal samples to obtain CFU/g feces. Data points represent mean plus SEM of $\mathrm{n}=3$ mice. (D) 
959 Enumerations of bacterial levels in cecal contents from the mice shown in C. (E) Expression of 960 the rus locus in wild-type $B t$ in the cecal contents from experiments in Figure 2A-B (main text) 961 and Figure S2A-B. Data points represent mean plus SEM of $n=4$ mice. Student's t-test was used

962 to compare all other samples to rus expression in 6-8 week old females on FR diet revealing no

963 significant (ns) differences. (F) GC/MS analysis of free and linked (acid-hydrolyzable)

964 monosaccharides in the FR and FF diets. Data is presented as mg of sugar per gram of diet. Bars 965 show the average plus SEM of $n=3$ technical replicates. Note that the FF diet contains $44 \%$ w/w 966 glucose, which is the source of the strong free glucose signal observed with that diet.

Figure S2. Monosaccharide content of mono-associated mice from cecal contents.

969 Data is related to Figure 2. GC/MS analysis of free and linked (acid-hydrolyzable)

970 monosaccharides from the cecal contents of wild type or $\Delta r u s$ mono-associated 6-8 week old,

971 female Swiss Webster mice maintained on a fiber rich diet for 42 days. Data is presented as $\mathrm{mg}$

972 of sugar per gram of cecal contents. The pink and olive green bars represent wild type linked and

973 free respectively, while the orange and green represent linked or free for the $\Delta r u s$ colonized

974 mice. Bars show the average plus SEM of $n=3$ technical replicates. N.D. indicates that the sugar

975 was not detectable above our limit of detection (L.O.D.) which is shown as a dotted line above

976 the ribose bars.

Figure S3. Rus deletion strains on ribose containing compounds.

979 Data is related to Figure 4. (A) Wild-type (maize, solid line) or $\Delta r u s K 1$ (blue, solid line) strains 980 grown in MM-ribose compared to wild-type and $\Delta r u s K 1$ strains that had been previously grown 981 on MM-ribose, isolated on solid medium (BHI-blood), two separate colonies picked into rich 982 media (TYG), and then grown in MM-ribose again shown as maize dashed lines (wild type) or 983 blue dashed line ( $\Delta r u s K 1)$ to check if the delayed growth phenotype associated with the $\Delta r u s K 1$ 984 strain was the product of a genetic suppressor mutation or similar epigenetic/reprogramming. (B-

985 F) Growth of rus deletion strains exhibiting similar or identical growth as wild-type on MM986 ribose (red) with no obvious growth defects. Growth on MM-glucose (black) is shown for 987 comparison. (G) Wild-type Bt grown in MM containing $5 \mathrm{mg} / \mathrm{ml}$ of one of the following 988 nucleosides (uridine, blue; cytidine, pink; 5-methyl uridine, green; or thymidine, purple) without 989 any ribose added. (H) Wild-type $B t$ grown on MM containing $5 \mathrm{mg} / \mathrm{ml}$ of yeast RNA without 
990 any exogenous enzymes. (I) rusC transcriptional response when wild-type $B t$ was exposed to

991 titrated amounts of ribose $(\mathrm{mg} / \mathrm{ml})$ where each data point represents a different 10 -fold dilution

992 of ribose. The red dot represents $0.5 \mathrm{mg} / \mathrm{ml}$ ribose which induces $r u s$ activation to comparable

993 levels as $5 \mathrm{mg} / \mathrm{ml}$ ribose the data point directly to the left of the red point compared to growth in

994 MM-glucose. (J) Wild-type growth on different concentrations of MM containing ribose at the

995 following concentrations (mg/ml): 5, black; 2.5, purple; 1.25, green; 0.625, orange; 0.5, red; or

9960.15 , blue. Growth was not detectable at levels $\leq 0.5 \mathrm{mg} / \mathrm{ml}$. (K) Wild-type growth on MM

997 containing nucleosides at concentrations of $5 \mathrm{mg} / \mathrm{ml}$ (solid lines) or $10 \mathrm{mg} / \mathrm{ml}$ (dashed lines) in

998 the presence of $0.5 \mathrm{mg} / \mathrm{ml}$ ribose. Individual nucleoside growths are colored same as (G). (L-P)

999 rus deletion strains exhibiting a complete lack of growth phenotype on all nucleosides tested are

1000 shown as labeled. (Q) Wild-type Bt does not grow detectably in MM containing the enzymes

1001 used for RNA degradation. (R-W) Individual mutants (as labeled) that do not show a growth

1002 defect on nucleosides in the presence of $0.5 \mathrm{mg} / \mathrm{ml}$ ribose.

1003

1004 Figure S4. Further characterization of nucleoside scavenging mutants and rus transcript 1005 activation dynamics, and relevance of global gene response to growth on ribose.

1006 Data is related to Figure 5. (A,B) Growth of NSS mutants or wild-type Bt on ribose (A) or RNA

1007 (B) (with added enzymes). Strains are color coded (wild-type, black; $\triangle B T 0184$, red; $\triangle B T 1881$,

1008 green; $\triangle B T 4330$, purple; or $\triangle B T 4554$, orange). (C) Multiple sequence alignment of BT2808

1009 (RusNH) and other RusNH-like proteins from Bacteroidetes (red boxed region) compared to

1010 previously validated nucleoside hydrolases isolated from bacteria (E. coli, RihA,B,C and $P$.

1011 fluorescens), archaea (S. solfataricus), parasitic eukaryotes (T. vivax, L. major, and C.

1012 fasciculata), moss (P. patens), maize (Zea mays), and yeast (S. pombe), indicating that the

1013 predicted nucleoside hydrolase of BT2808 shares the universally conserved N-terminal

1014 DXDXXXDD motif responsible for $\mathrm{Ca}^{2+}$ coordination $\left(2^{\text {nd }}\right.$ and $4^{\text {th }}$ yellow-highlighted aspartic

1015 acid residues) and ribose binding ( $3^{\text {rd }}$ yellow-highlighted aspartic acid), as well as the nearly

1016 conserved canonical $1^{\text {st }}$ aspartic acid residue denoting the motif (yellow or green highlighted

1017 position). Specific to the Bacteroidetes nucleoside hydrolases, there are two additional conserved

1018 residues, an asparagine and an additional aspartic acid within the motif (highlighted in teal) not

1019 found IUNH family nucleoside hydrolases outside of the Bacteroidetes. (D) Immunofluorescent

1020 microscopy of intact Bt whole cells grown in MM-ribose media staining with anti-BT2807 
1021 (RusGH) antibody, indicating that the protein is localized to the outer surface as the secondary

1022 antibody has clearly labeled nearly all of the cells seen in the brightfield image (left), a green

1023 color in the fluorescent image at (right). (E) Expression of BT2804 and BT2805 transcript in

1024 wild-type $B t$ (purple) or $\Delta r u s K 1$ (red) strains after active growth had initiated in MM-ribose and

1025 cells were sampled in mid-log phase and compared to growth in MM-glucose. Bars represent

1026 mean, plus SD of $\mathrm{n}=3$ replicates. (F) rus transcript during a time course experiment in which

1027 cells were shifted from growth on MM-glucose to MM-ribose and transcript measured over time

1028 with points every 5 minutes for the first 30 minutes and every 15 minutes after out to the

1029 conclusion at 120 minutes post-ribose exposure. For the wild-type (black) and $\Delta r u s D$ (dark

1030 yellow) strains, the rusC gene was probed, while for the $\Delta r u s C$ strain (red), the rusD gene was

1031 probed (similar kinetics were seen in the wild-type strain when the rusD gene was used to assay

1032 rus activation, data not shown). Error bars represent the SEM of $\mathrm{n}=3$ replicates performed on

1033 separate days. (G) Similar to the response experiment in Figure 5E but using nucleosides

1034 (inosine, black line or uridine, green line) and probing rusC expression to address if either a

1035 catabolized (uridine) or non-catabolized (inosine) ribose containing compound could stimulate

1036 rus activation, with no response detected compared to growth in MM-glucose. $(\mathrm{H})$ As in Figure

$10375 \mathrm{~F}$, we probed expression of the genes required for xylose metabolism (BT0791-0795) when

1038 wild-type $B t$ was grown on MM-xylose (green bars) or MM-ribose (blue bars) and compared to

$1039 B t$ grown in MM-glucose. (I) in vivo competition of a strain lacking the entire fructan PUL,

1040 BT1754-1765 ( $\triangle$ fruc, blue line) against a strain lacking both the fructan PUL and the rus PUL

1041 ( $\Delta$ fruc/drus, orange line) in 6-8 week old Swiss-Webster female mice maintained on the FR diet.

1042 The relative fecal abundance is shown on a log scale as assayed by qPCR over the course of the

1043 experiment, error bars show the SEM of $n=4$ mice. $(J)$ in vivo rus expression from cecal contents

1044 of the mice in I. measuring expression of the $r u s C$ gene, error bar shows the SEM of $\mathrm{n}=4 \mathrm{mice}$.

1045

1046 Figure S5. An expanded repertoire of rus architectures across the Bacteroidetes phylum.

1047 Related to Figure 6. Comparative genomics analysis of a broader survey of members of the

1048 Bacteroidetes phylum, revealing many different types of the rus locus. This figure displays

1049 almost all of the additional locus types found in both the human gut isolates along with those

1050 found in aquatic, soil, and human oral environments. Not shown are subtypes, where the same

1051 genes are present, but arranged differently, as well as types 21, 30, 33, 36, 39, 44, 48, 46, and 61, 
1052 for which only one example was identified and gene arrangements had less complexity than the

1053 majority shown here (all loci are listed in Table S5). As in Figure 6, the gene size is scaled

1054 within and between genomes and the background color is kept constant for genes predicted to

1055 encode the same functions. Gene abbreviations are as follows in order of appearance: (DeoR,

1056 DeoR-like family of transcriptional regulator; MFS, Major Facilitator Superfamily of

1057 transporters; FADOR, Flavin (FAD) Oxidoreductase; NAD, NAD Binding Protein; CK,

1058 Carbohydrate Kinase, unknown family; GDPDE, Glycerophosphoryl Diester Phosphodiesterase;

1059 NH, Nucleoside Hydrolase; GH, Glycoside hydrolase; HAD, Haloacid Dehydrogenase; LacI,

1060 LacI-type transcriptional regulator; FrcK, fructokinase; FGE, Formylglycine-Generating

1061 Enzyme, required for sulfatase activity; NADP-DH, NADP-Dependent aldehyde

1062 Dehydrogenase; ALT-DH, Altronate Dehydrogenase; kdxD, 2-dehydro-3-deoxy-D-arabinonate

1063 dehydratase; AraC, AraC-like transcriptional regulator; Rib Iso, Ribose-5-Phosphate Isomerase;

1064 Tn-ase, transposase; BACON, Bacteroidetes-Associated Carbohydrate-binding Often N-terminal

1065 domain; cpdA, 3'.5'-cyclic AMP phosphodiesterase; EEPase, Endo-Exo Nucleoside-

1066 Phosphatase; TAT, Twin-Arginine Translocase; BNR, BNR repeat-like domain; SIAE, Sialate

1067 O-acetylesterase; DPP IV, Dipeptidyl-peptidase IV; ***, RNA polymerase sigma factor ECF

1068 subfamily; FecR, FecR-like transcriptional regulator; SusE, Bacteroides SusE-like outer

1069 membrane binding protein; GntR, GntR-like transcriptional regulator; FBA, Fructose

1070 Bisphosphate Aldolase; Xyl Iso, Xylose Isomerase; ROK, Repressor/ORF/Kinase domain

1071 containing protein; ADH, Alcohol Dehydrogenase; LmbE, N-acetylglucosaminyl deacetylase

1072 LmbE-like family; E/L/P, Esterase/Lipase/Peptidase-like domain containing protein; RhaA,

1073 Regulator of RNaseE activity; Acid Pase, Acid Phosphotase-like protein).

1074

1075 References

1076

1077

1078

1079

Bjursell, M.K., Martens, E.C., and Gordon, J.I. (2006). Functional genomic and metabolic studies of the adaptations of a prominent adult human gut symbiont, Bacteroides thetaiotaomicron, to the suckling period. J. Biol. Chem. 281, 36269-36279.

1080 Cameron, E.A., Kwiatkowski, K.J., Lee, B.H., Hamaker, B.R., Koropatkin, N.M., and Martens, 1081 E.C. (2014). Multifunctional nutrient-binding proteins adapt human symbiotic bacteria for 1082 glycan competition in the gut by separately promoting enhanced sensing and catalysis. mBio 5 . 1083 e01441-14 
1084 Cuskin, F., Lowe, E.C., Temple, M.J., Zhu, Y., Cameron, E., Pudlo, N.A., Porter, N.T., Urs, K., 1085 Thompson, A.J., Cartmell, A., et al. (2015). Human gut Bacteroidetes can utilize yeast mannan 1086 through a selfish mechanism. Nature 517, 165-169.

1087 Datsenko, K.A., and Wanner, B.L. (2000). One-step inactivation of chromosomal genes in 1088 Escherichia coli K-12 using PCR products. Proc. Natl. Acad. Sci. USA 97, 6640-6645.

1089 David, L.A., Maurice, C.F., Carmody, R.N., Gootenberg, D.B., Button, J.E., Wolfe, B.E., Ling, 1090 A.V., Devlin, A.S., Varma, Y., Fischbach, M.A., et al. (2014). Diet rapidly and reproducibly 1091 alters the human gut microbiome. Nature 505, 559-563.

1092 Desai, M.S., Seekatz, A.M., Koropatkin, N.M., Kamada, N., Hickey, C.A., Wolter, M., Pudlo, 1093 N.A., Kitamoto, S., Terrapon, N., Muller, A., et al. (2016). A Dietary Fiber-Deprived Gut 1094 Microbiota Degrades the Colonic Mucus Barrier and Enhances Pathogen Susceptibility. Cell 1095 167, 1339-1353 e1321.

1096 Duquesne, S., Petit, V., Peduzzi, J., and Rebuffat, S. (2007). Structural and functional diversity 1097 of microcins, gene-encoded antibacterial peptides from enterobacteria. J. Mol. Microb. Biotech. 1098 13, 200-209.

1099 Fabich, A.J., Jones, S.A., Chowdhury, F.Z., Cernosek, A., Anderson, A., Smalley, D., 1100 McHargue, J.W., Hightower, G.A., Smith, J.T., Autieri, S.M., et al. (2008). Comparison of 1101 Carbon Nutrition for Pathogenic and Commensal Escherichia coli Strains in the Mouse Intestine. 1102 Infect. Immun. 76, 1143-1152.

1103 Finkel, S.E., and Kolter, R. (2001). DNA as a nutrient: novel role for bacterial competence gene 1104 homologs. J. Bacteriol. 183, 6288-6293.

1105 Flint, H.J., Scott, K.P., Duncan, S.H., Louis, P., and Forano, E. (2012). Microbial degradation of 1106 complex carbohydrates in the gut. Gut Microbes 3, 289-306.

1107 Glenwright, A.J., Pothula, K.R., Bhamidimarri, S.P., Chorev, D.S., Basle, A., Firbank, S.J., 1108 Zheng, H., Robinson, C.V., Winterhalter, M., Kleinekathofer, U., et al. (2017). Structural basis 1109 for nutrient acquisition by dominant members of the human gut microbiota. Nature 541, 4071110411.

1111 Goodman, B.E. (2010). Insights into digestion and absorption of major nutrients in humans. Adv. 1112 Physiol. Educ. 34, 44-53.

1113 Hackland, L.P., Parolis, H., and Parolis, L.A.S. (1991). Escherichia coli O9:K38 capsular 1114 antigen: another ribofuranose-containing glycan. Carbohyd. Res. 219, 193-201.

1115 Hammer-Jespersen, K., Munch-Petersen, A., Schwartz, M., and Nygaard, P. (1971). Induction of 1116 Enzymes Involved in the Catabolism of Deoxyribonucleosides and Ribonucleosides in E.coli 1117 K12, 1971. Eur. J. Biochem. 19, 533-538.

1118 Harvey, P.C., Watson, M., Hulme, S., Jones, M.A., Lovell, M., Berchieri, A., Jr., Young, J., 1119 Bumstead, N., and Barrow, P. (2011). Salmonella enterica serovar typhimurium colonizing the 
1120 lumen of the chicken intestine grows slowly and upregulates a unique set of virulence and 1121 metabolism genes. Infect. Immun. 79, 4105-4121.

1122 Hehemann, J.K., A.G.; Pudlo, P.A.; Martens, E.C.; Boraston, A.B. (2012). Bacteria of the human 1123 gut microbiome catabolize seaweed glycans with carbohydrate-active enzymes update from 1124 extrinsic microbes. Proc. Natl. Acad. Sci. USA 109, 19786-19791.

1125 Holdeman, L.V.C. (1977). Anaerobe Laboratory Manual, 4th edn. (Anaerobe Laboratory 1126 Virginia Polytechnic Institute \& State University).

1127 Iqbal, J., and Hussain, M.M. (2009). Intestinal lipid absorption. Am. J. Physiol-Endoc. M. 296, 1128 e1183-1194.

1129 Kim, H.S., Lee, J.H., Lee, W.S., and Bang, W.G. (2006). Genes encoding ribonucleoside 1130 hydrolase 1 and 2 from Corynebacterium ammoniagenes. Microbiology 152, 1169-1177.

1131 Knirel, Y.A., Kocharova, N.A., Bystrova, O.V., Katzenellenbogen, E., and Gamian, A. (2002). 1132 Structures and serology of the O-specific polysaccharides of bacteria of the genus Citrobacter. 1133 Arch. Immunol. Ther. Exp. 50, 379-391.

1134 Koropatkin, N.M., Martens, E.C., Gordon, J.I., and Smith, T.J. (2008). Starch catabolism by a 1135 prominent human gut symbiont is directed by the recognition of amylose helices. Structure 16, $1136 \quad 1105-1115$.

1137 Larsbrink, J., Rogers, T.E., Hemsworth, G.R., McKee, L.S., Tauzin, A.S., Spadiut, O., Klinter, 1138 S., Pudlo, N.A., Urs, K., Koropatkin, N.M., et al. (2014). A discrete genetic locus confers 1139 xyloglucan metabolism in select human gut Bacteroidetes. Nature 506, 498-502.

1140 Liang, L., He, X., Liu, G., and Tan, H. (2008). The role of a purine-specific nucleoside hydrolase 1141 in spore germination of Bacillus thuringiensis. Microbiology 154, 1333-1340.

1142 Luis, A.S., Briggs, J., Zhang, X., Farnell, B., Ndeh, D., Labourel, A., Basle, A., Cartmell, A., 1143 Terrapon, N., Stott, K., et al. (2018). Dietary pectic glycans are degraded by coordinated enzyme 1144 pathways in human colonic Bacteroides. Nat. Microbiol. 3, 210-219.

1145 Macfarlane, S., and Macfarlane, G.T. (2003). Regulation of short-chain fatty acid production. 1146 Proc. Nutr. Soc. 62, 67-72.

1147 Maltby, R., Leatham-Jensen, M.P., Gibson, T., Cohen, P.S., and Conway, T. (2013). Nutritional 1148 basis for colonization resistance by human commensal Escherichia coli strains HS and Nissle 11491917 against E. coli $\mathrm{O} 157: \mathrm{H} 7$ in the mouse intestine. PLoS One 8, e53957.

1150 Martens, E.C., Chiang, H.C., and Gordon, J.I. (2008). Mucosal glycan foraging enhances fitness 1151 and transmission of a saccharolytic human gut bacterial symbiont. Cell Host Microbe 4, 447-457.

1152 Martens, E.C., Koropatkin, N.M., Smith, T.J., and Gordon, J.I. (2009). Complex glycan 1153 catabolism by the human gut microbiota: the Bacteroidetes Sus-like paradigm. J. Biol. Chem. $1154 \quad 284,24673-24677$. 
1155 Martens, E.C., Lowe, E.C., Chiang, H., Pudlo, N.A., Wu, M., McNulty, N.P., Abbott, D.W., 1156 Henrissat, B., Gilbert, H.J., Bolam, D.N., et al. (2011). Recognition and degradation of plant cell 1157 wall polysaccharides by two human gut symbionts. PLoS Biol. 9, e1001221.

1158 Martinez-Jehanne, V., du Merle, L., Bernier-Febreau, C., Usein, C., Gassama-Sow, A., Wane, 1159 A.A., Gouali, M., Damian, M., Aidara-Kane, A., Germani, Y., et al. (2009). Role of deoxyribose 1160 catabolism in colonization of the murine intestine by pathogenic Escherichia coli strains. Infect. 1161 Immun. 77, 1442-1450.

1162 McConnell, R.E., Higginbotham, J.N., Shifrin, D.A., Jr., Tabb, D.L., Coffey, R.J., and Tyska, 1163 M.J. (2009). The enterocyte microvillus is a vesicle-generating organelle. J. Cell. Biol. 185, 1164 1285-1298.

1165 McLeod, A., Snipen, L., Naterstad, K., and Axelsson, L. (2011). Global transcriptome response 1166 in Lactobacillus sakei during growth on ribose. BMC Microbiol. 11, 145.

1167 Nakjang, S., Ndeh, D.A., Wipat, A., Bolam, D.N., and Hirt, R.P. (2012). A novel extracellular 1168 metallopeptidase domain shared by animal host-associated mutualistic and pathogenic microbes. 1169 PloS One 7, e30287.

1170 Ndeh, D., Rogowski, A., Cartmell, A., Luis, A.S., Basle, A., Gray, J., Venditto, I., Briggs, J., 1171 Zhang, X., Labourel, A., et al. (2017). Complex pectin metabolism by gut bacteria reveals novel 1172 catalytic functions. Nature 544, 65-70.

1173 Palchevskiy, V., and Finkel, S.E. (2009). A role for single-stranded exonucleases in the use of 1174 DNA as a nutrient. J. Bacteriol. 191, 3712-3716.

1175 Pan, N., and Imlay, J.A. (2001). How does oxygen inhibit central metabolism in the obligate 1176 anaerobe Bacteroides thetaiotaomicron. Mol. Microbiol. 39, 1562-1571.

1177 Parkin, D.W., Horenstein, B.A., Abdulah, D.R., Estupinan, B., and Schramm, V.L. (1991). 1178 Nucleoside Hydrolase from Crithidia fasciculata. Metabolic role, purification, specificity, and 1179 kinetic mechanism. J. Biol. Chem. 266, 20658-20665.

1180 Petersen, C., and Moller, L.B. (2001). The RihA, RihB, and RihC ribonucleoside hydrolases of 1181 Escherichia coli. Substrate specificity, gene expression, and regulation. J. Biol. Chem. 276, 8841182894.

1183 Pettolino, F.A., Walsh, C., Fincher, G.B., and Bacic, A. (2012). Determining the polysaccharide 1184 composition of plant cell walls. Nat. Protoc. 7, 1590-1607.

1185 Pluvinage, B., Grondin, J.M., Amundsen, C., Klassen, L., Moote, P.E., Xiao, Y., Thomas, D., 1186 Pudlo, N.A., Anele, A., Martens, E.C., et al. (2018). Molecular basis of an agarose metabolic 1187 pathway acquired by a human intestinal symbiont. Nat. Commun. 9, 1043.

1188 Pokusaeva, K., Neves, A.R., Zomer, A., O'Connell-Motherway, M., MacSharry, J., Curley, P., 1189 Fitzgerald, G.F., and van Sinderen, D. (2010). Ribose utilization by the human commensal 1190 Bifidobacterium breve UCC2003. Microb. Biotechnol. 3, 311-323. 
1191 Porter, N.T., and Martens, E.C. (2017). The Critical Roles of Polysaccharides in Gut Microbial 1192 Ecology and Physiology. Annu. Rev. Microbiol. 71, 349-369.

1193 Pudlo, N.A., Urs, K., Kumar, S.S., German, J.B., Mills, D.A., and Martens, E.C. (2015).

1194 Symbiotic Human Gut Bacteria with Variable Metabolic Priorities for Host Mucosal Glycans.

1195 mBio 6, e01282-01215.

1196 Renzi, F., Manfredi, P., Dol, M., Fu, J., Vincent, S., and Cornelis, G.R. (2015). Glycan-foraging 1197 systems reveal the adaptation of Capnocytophaga canimorsus to the dog mouth. mBio 6, e02507.

1198 Schlimme, E., Martin, D., and Meisel, H. (2000). Nucleosides and Nucleotides; Natural 1199 Bioactive Substances in Milk and Colostrum. Br. J. Nutr. 84, S59-68.

1200 Shi, W., Schramm, V.L., and Almo, S.C. (1999). Nucleoside Hydrolase from Leishmania major: 1201 Cloning, Expression, Catalytic Properties, Transition State Inhibitors, And The 2.5-A Crystal 1202 Structure. J. Biol. Chem. 274, 21114-21120.

1203 Sonnenburg, E.D., Smits, S.A., Tikhonov, M., Higginbottom, S.K., Wingreen, N.S., and 1204 Sonnenburg, J.L. (2016). Diet-induced extinctions in the gut microbiota compound over 1205 generations. Nature 529, 212-215.

1206 Sonnenburg, E.D., Zheng, H., Joglekar, P., Higginbottom, S.K., Firbank, S.J., Bolam, D.N., and 1207 Sonnenburg, J.L. (2010). Specificity of polysaccharide use in intestinal Bacteroides species 1208 determines diet-induced microbiota alterations. Cell 141, 1241-1252.

1209 Sonnenburg, J.L., Xu, J., Leip, D.D., Chen, C.H., Westover, B.P., Weatherford, J., Buhler, J.D., 1210 and Gordon, J.I. (2005). Glycan foraging in vivo by an intestine-adapted bacterial symbiont. 1211 Science 307, 1955-1959.

1212 Temple, M.J., Cuskin, F., Basle, A., Hickey, N., Speciale, G., Williams, S.J., Gilbert, H.J., and 1213 Lowe, E.C. (2017). A Bacteroidetes locus dedicated to fungal 1,6-beta-glucan degradation: 1214 Unique substrate conformation drives specificity of the key endo-1,6-beta-glucanase. J. Biol. 1215 Chem. 292, 10639-10650.

1216 TerAvest, M.H., Z; Rosenbaum, MA; Martens, EC; Cotta, MA; Gordon, JI; Angenent, LT 1217 (2013). Regulated Expression of Polysaccharide Utilization and Capsular Biosynthesis Loci in 1218 Biofilm and Planktonic Bacteroides thetaiotamicron During Growth in Chemostats. Biotechnol. 1219 Bioeng. 111, 165-173.

1220 Terrapon, N., Lombard, V., Drula, E., Lapebie, P., Al-Masaudi, S., Gilbert, H.J., and Henrissat, 1221 B. (2018). PULDB: the expanded database of Polysaccharide Utilization Loci. Nucleic Acids 1222 Res. 46, D677-D683.

1223 van Arkel, J., Sevenier, R., Hakkert, J.C., Bouwmeester, H.J., Koops, A.J., and van der Meer, 1224 I.M. (2013). Tailor-made fructan synthesis in plants: a review. Carbohydr. Polym. 93, 48-56.

1225 Versées, W., and Steyaert, J. (2003). Catalysis by nucleoside hydrolases. Curr. Opin. Struct. 1226 Biol. 13, 731-738. 
1227 Weickmann, J.L., Olson, E.M., and Glitz, D.G. (1984). Immunological Assay of Pancreatic 1228 Ribonucleases in Serum as an Indicator of Pancreatic Cancer. Cancer Res. 44, 1682-1687.

1229 Zimmermann, M., Zimmermann-Kogadeeva, M., Wegmann, R., and Goodman, A.L. (2019). 1230 Separating host and microbiome contributions to drug pharmacokinetics and toxicity. Science 1231363 , eaat9931. 


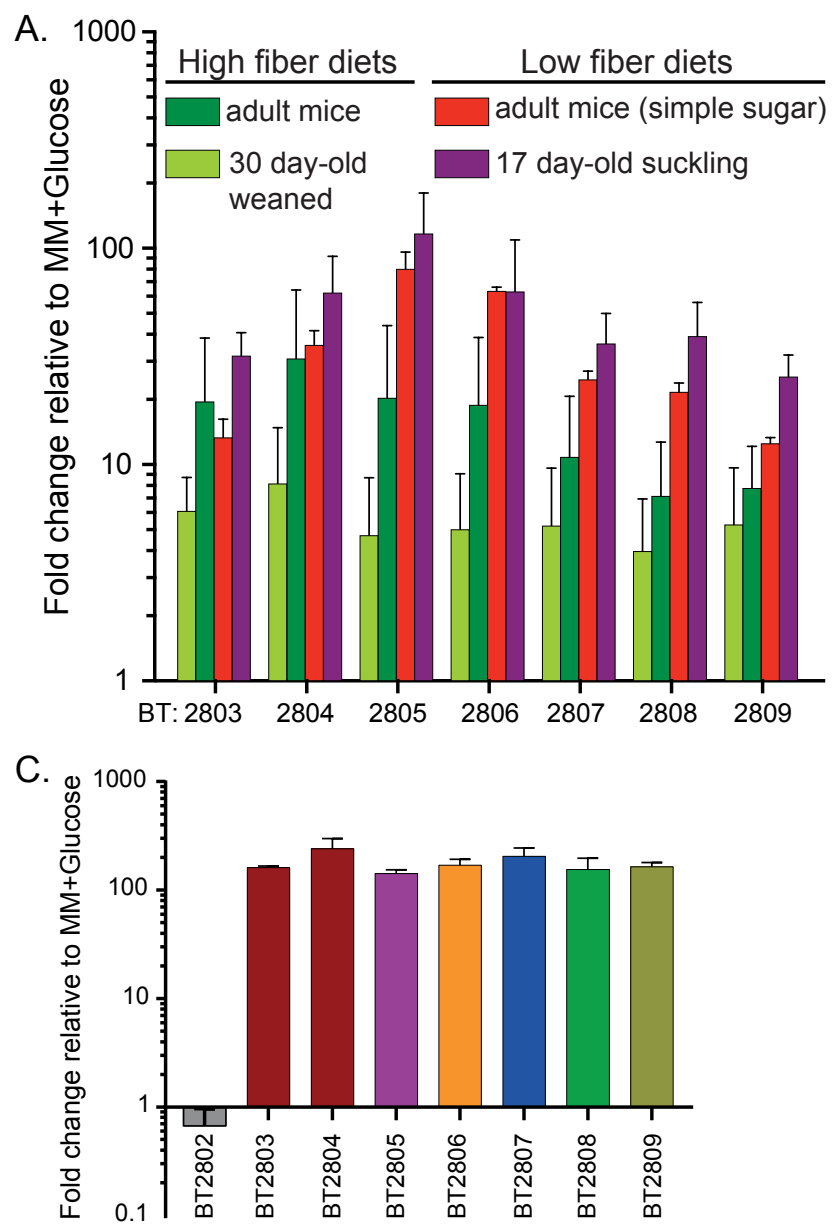

B.

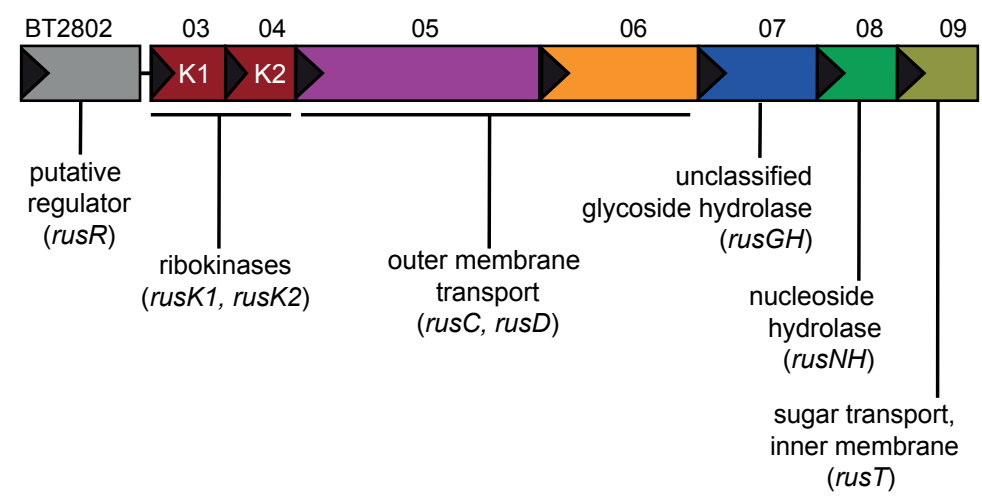

D.

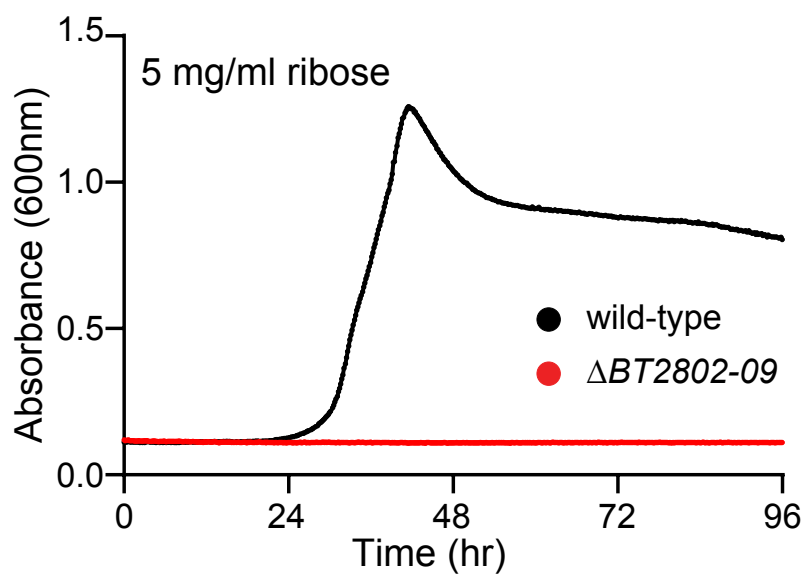


A.

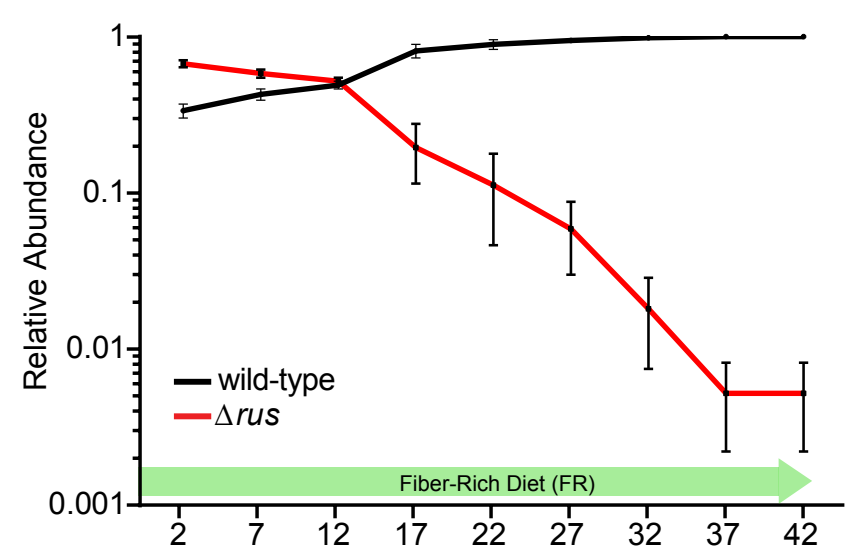

C.

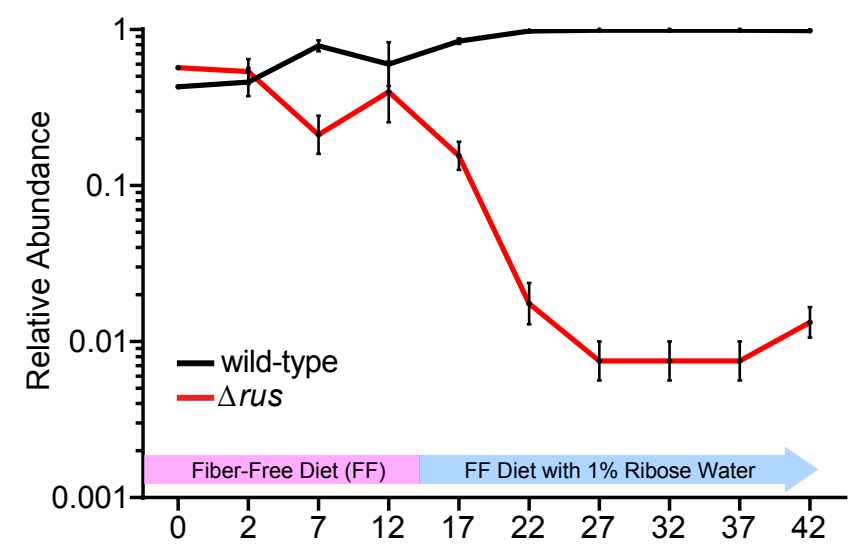

E.

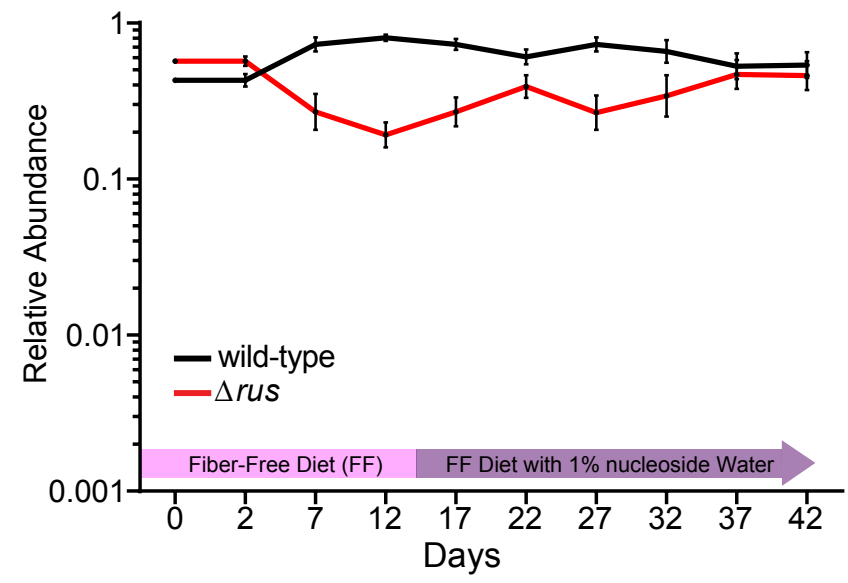

B.

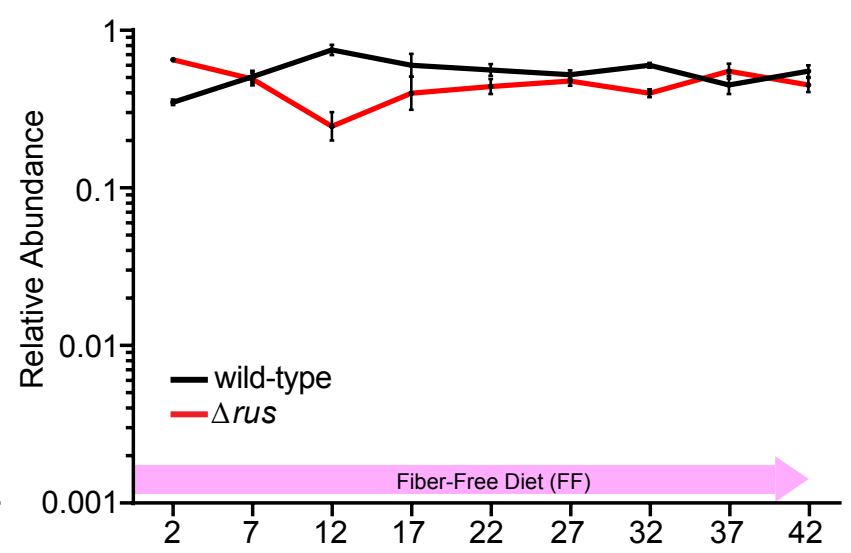

D.

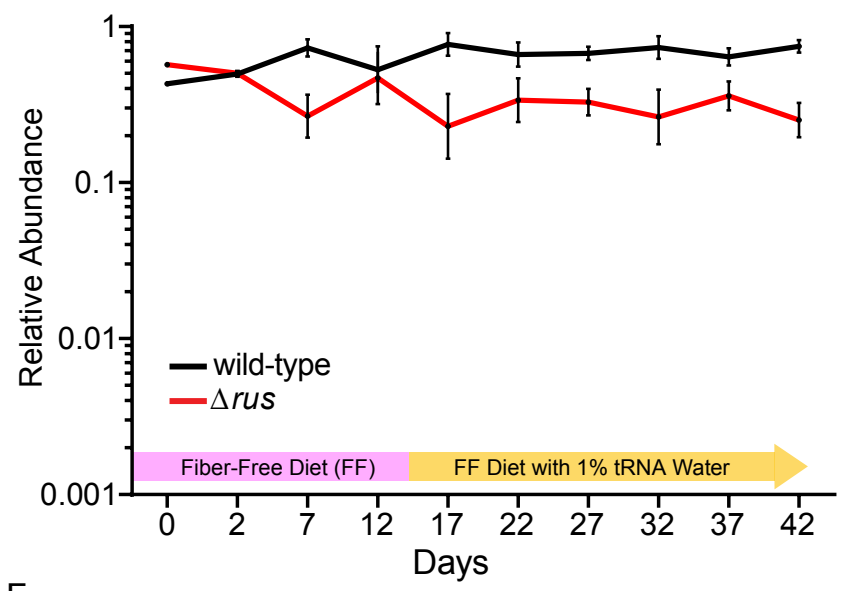

F.

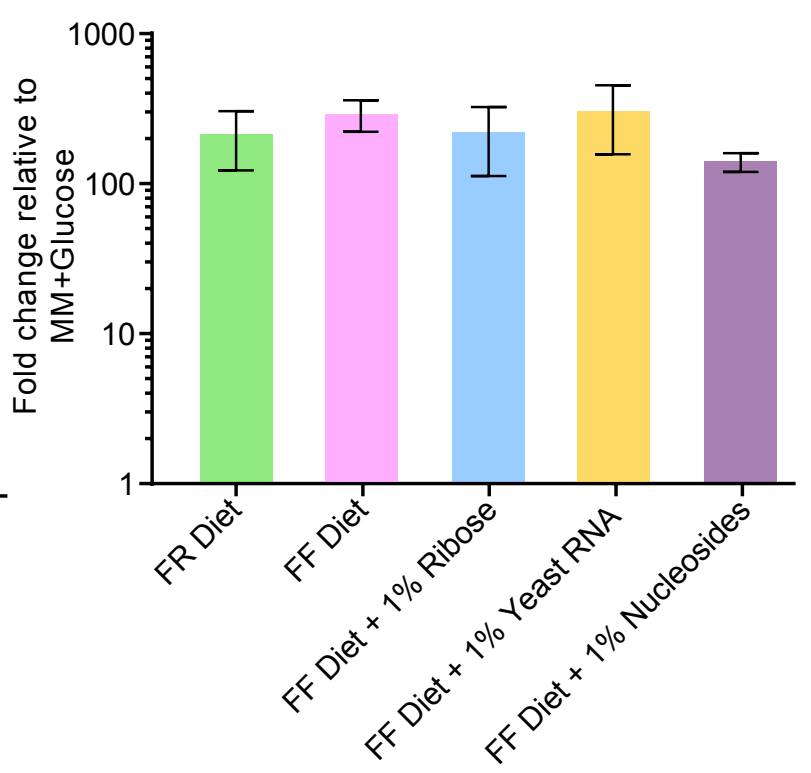



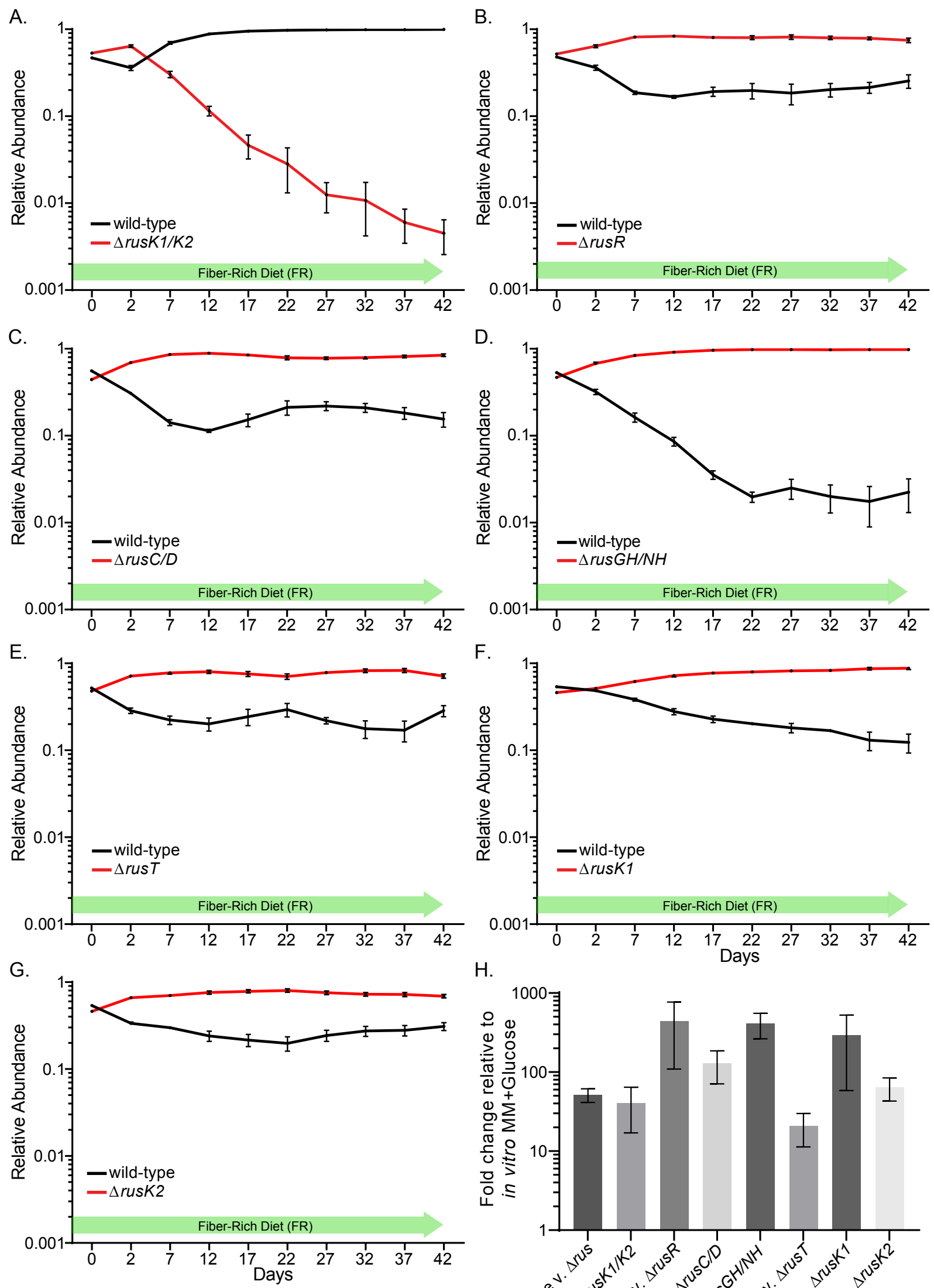

H.

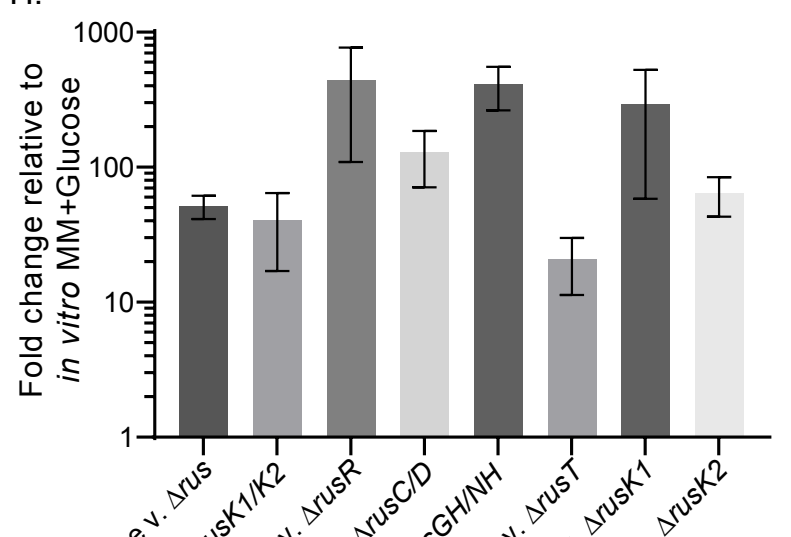


Glowacki et al. Figure 4
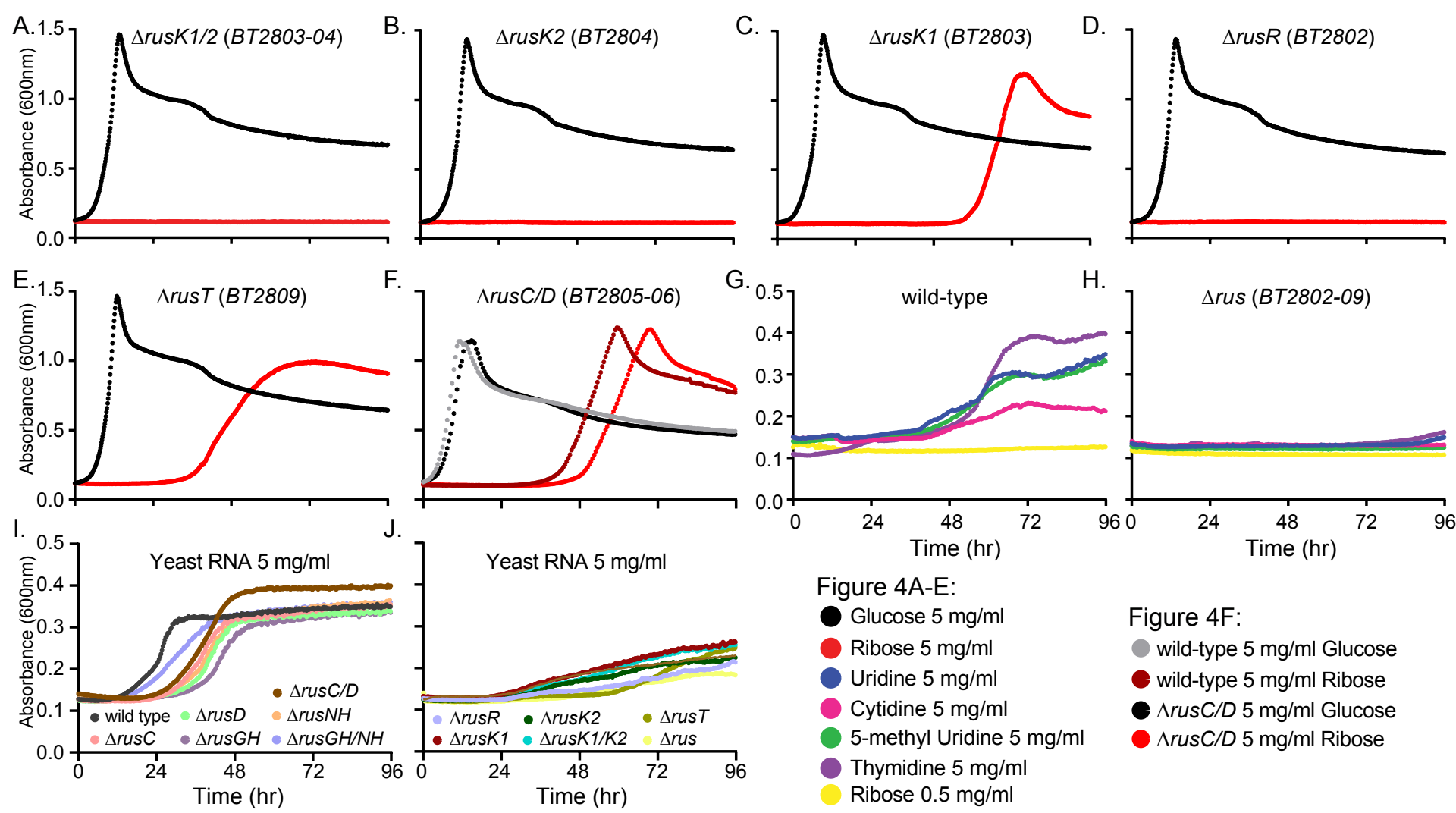

Figure 4A-E:

Glucose $5 \mathrm{mg} / \mathrm{ml}$

Ribose $5 \mathrm{mg} / \mathrm{ml}$

Uridine $5 \mathrm{mg} / \mathrm{ml}$

Cytidine $5 \mathrm{mg} / \mathrm{ml}$

5 -methyl Uridine $5 \mathrm{mg} / \mathrm{ml}$

Thymidine $5 \mathrm{mg} / \mathrm{ml}$

Ribose $0.5 \mathrm{mg} / \mathrm{ml}$

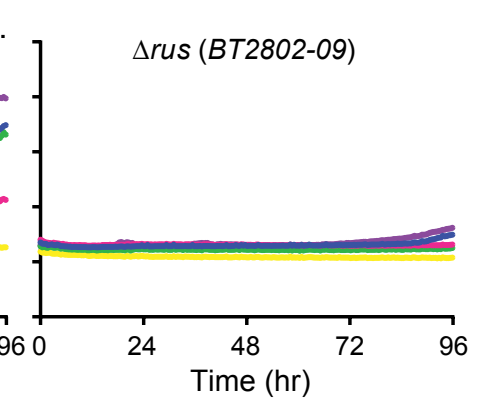

Figure 4F:

wild-type $5 \mathrm{mg} / \mathrm{ml}$ Glucose

- wild-type $5 \mathrm{mg} / \mathrm{ml}$ Ribose

- $\Delta r u s C / D 5 \mathrm{mg} / \mathrm{ml}$ Glucose

- $\Delta r u s C / D 5$ mg/ml Ribose 
A.

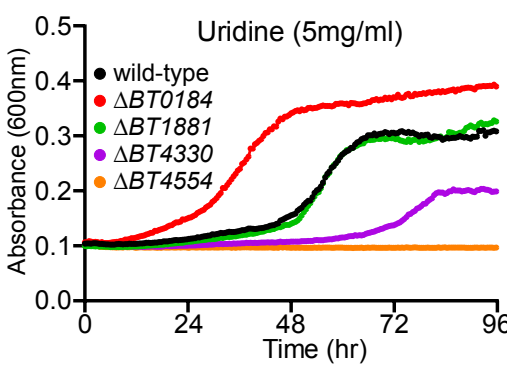

E.

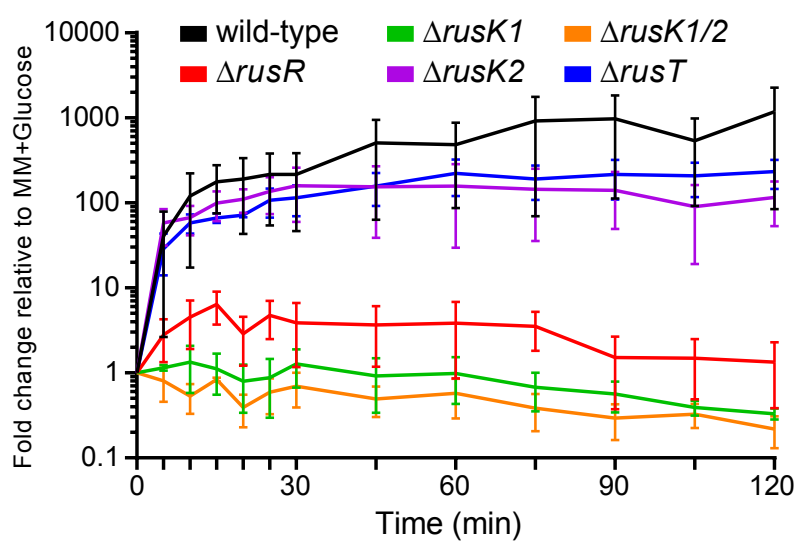

C.

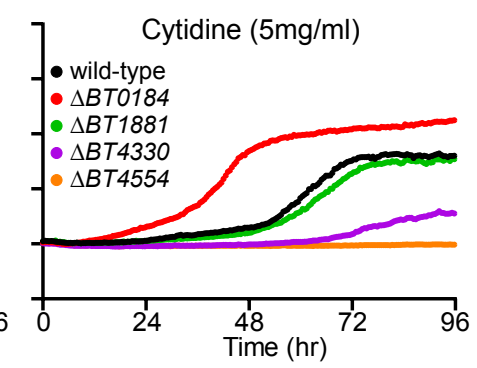

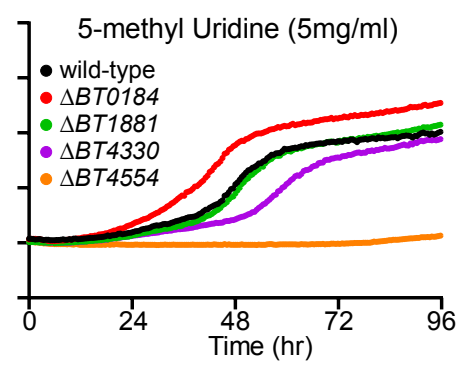

D.

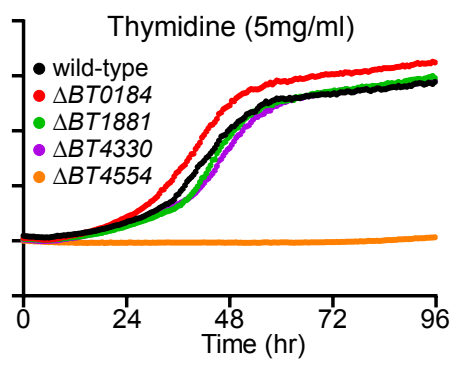

G.

F.

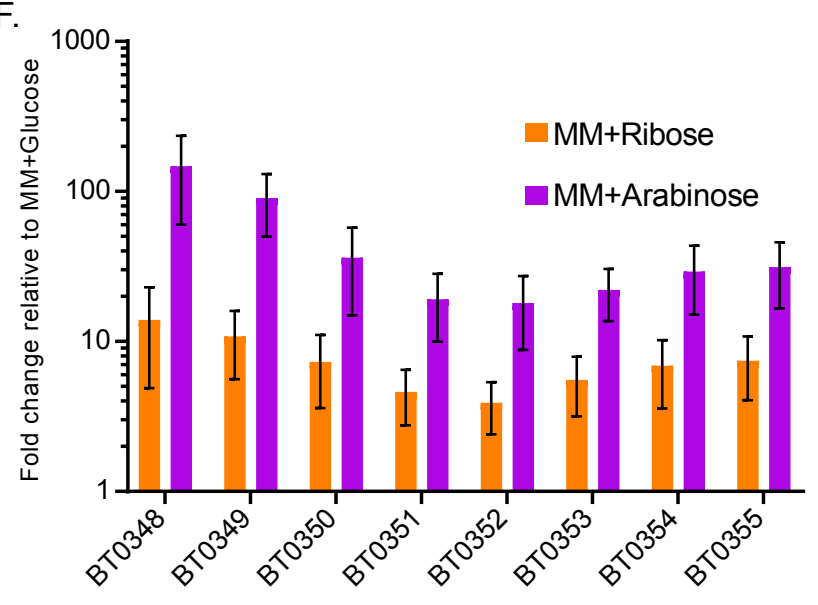

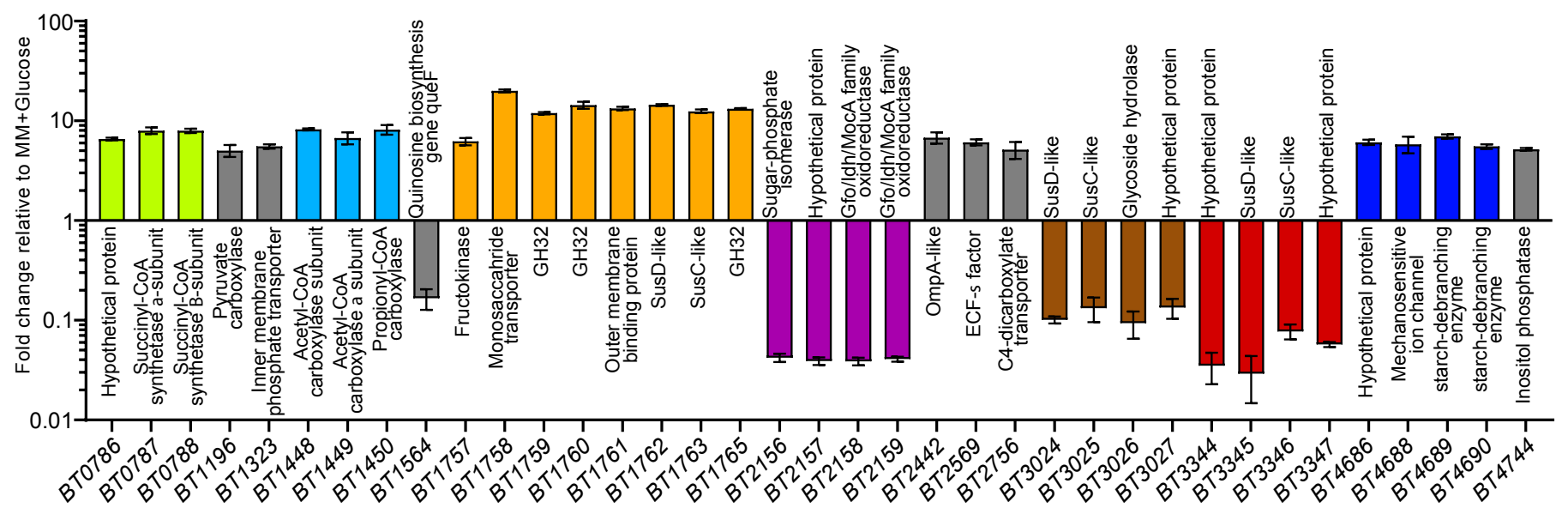


A.

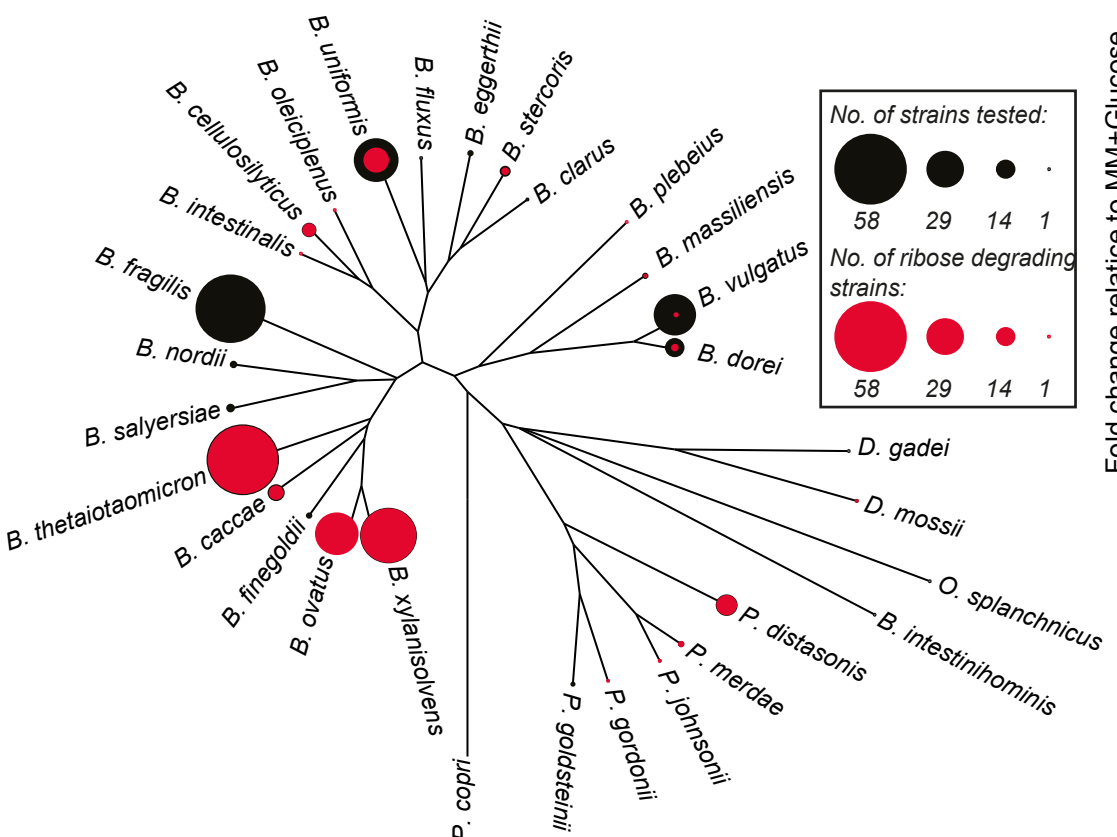

C.

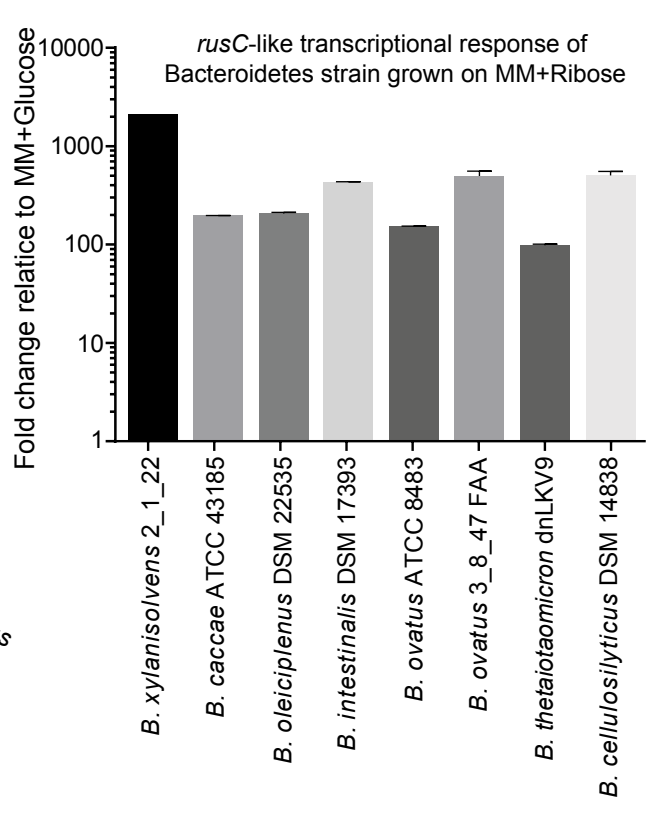

B.

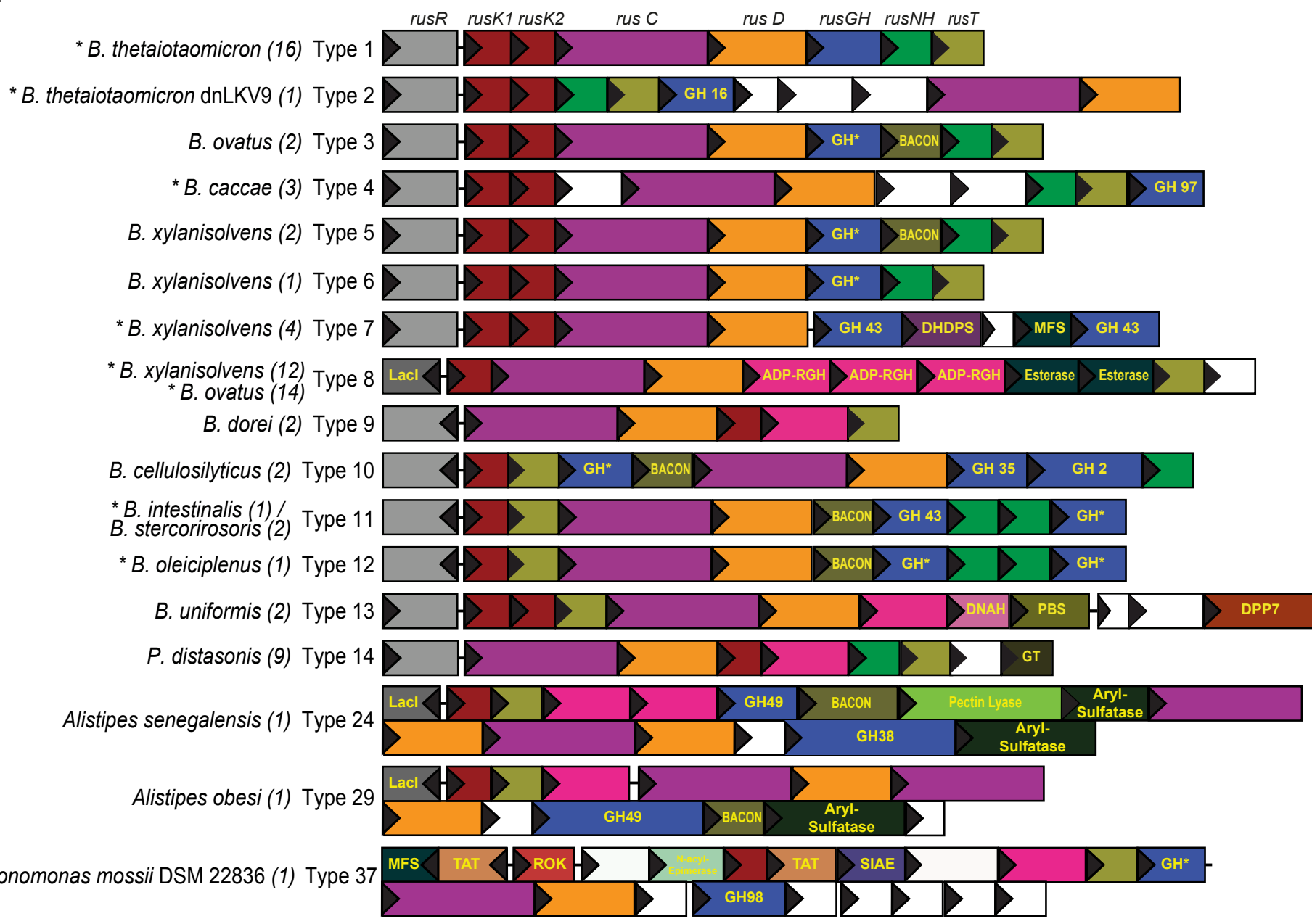




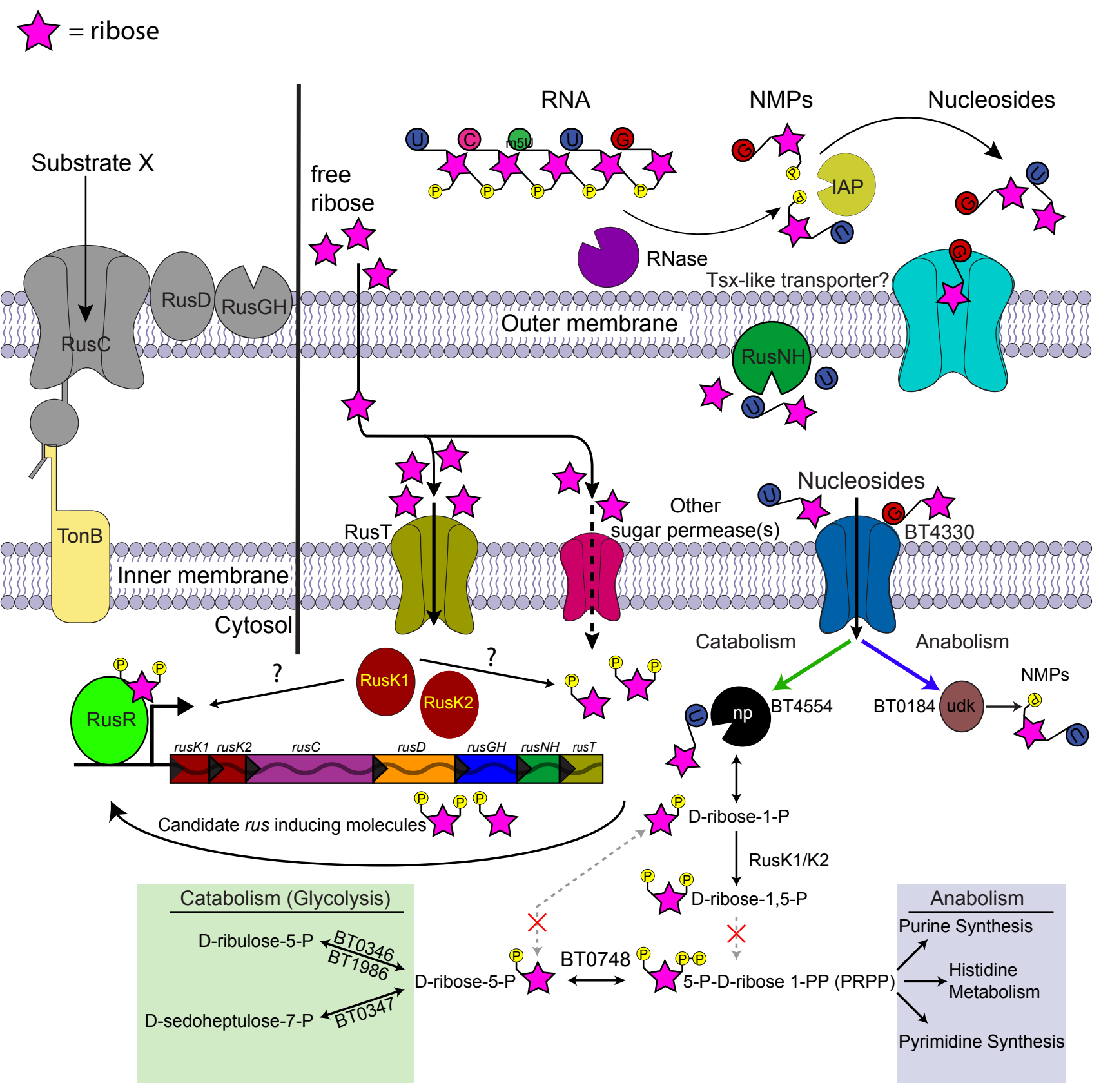




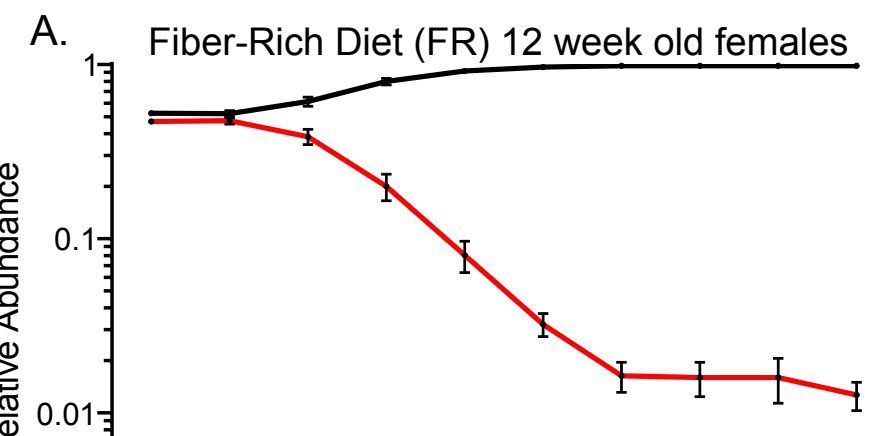

B.

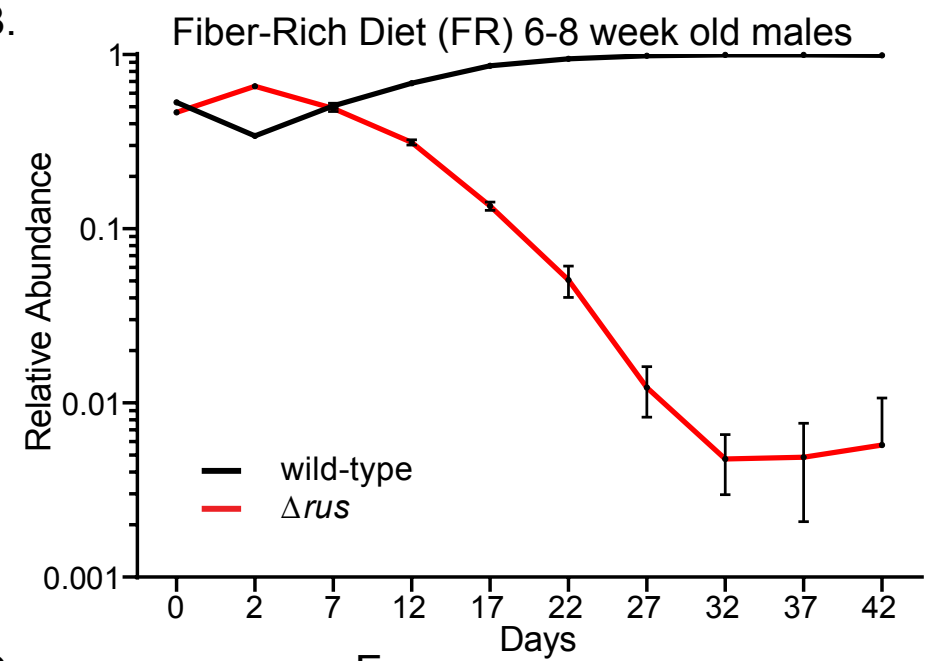

C.

- wild-type

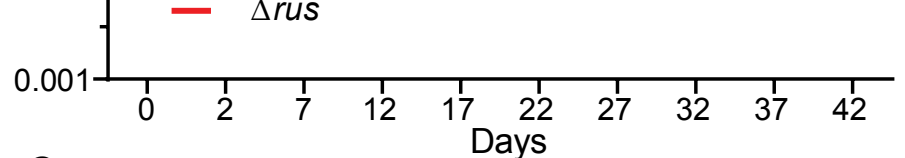
$10^{12}$

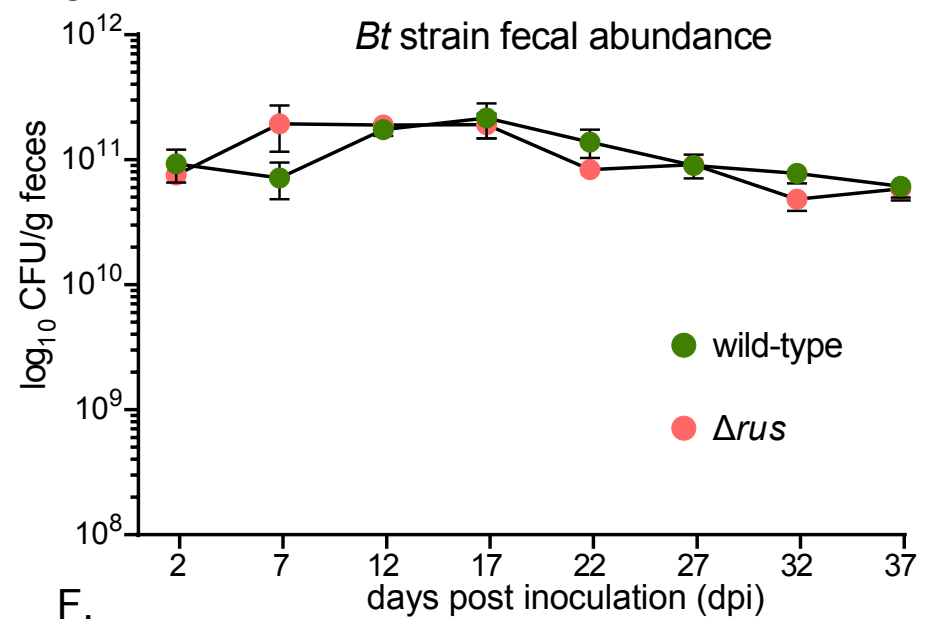

F.

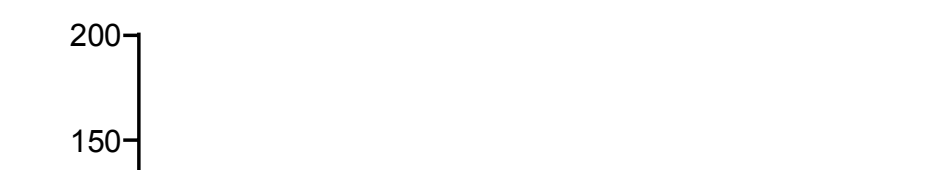

迹 $100-$

तิ $25 \mathrm{~T}$

节

Fiber-Rich, acid hydrolyzed

- Fiber-Free, acid hydrolyzed

Fiber-Rich, free sugars

Fiber-Free, free sugars

D.

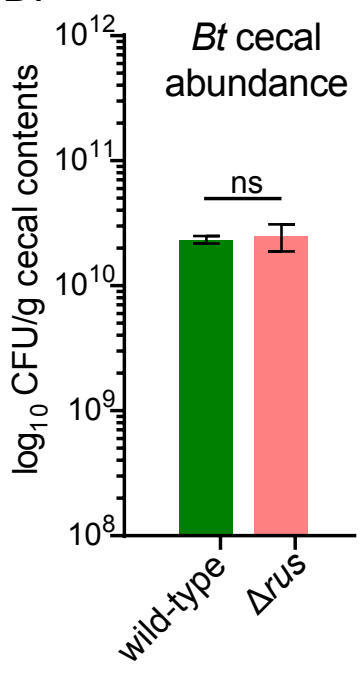

E.

in vivo cecal rus expression (BT2805)
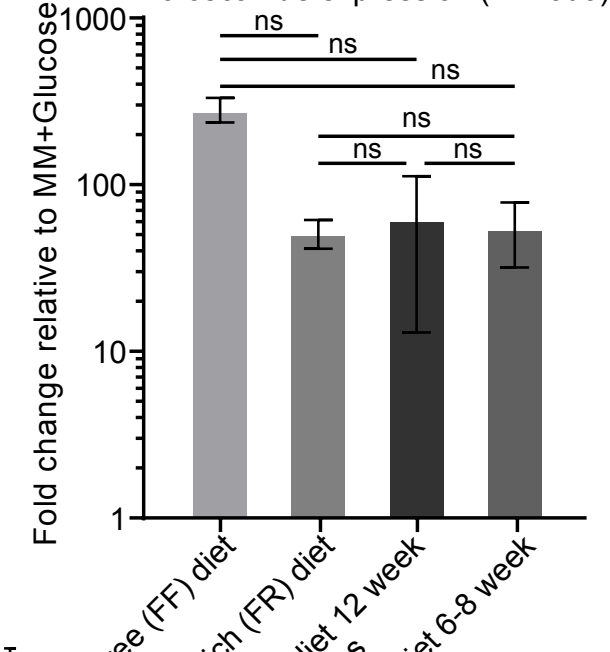
Glowacki et al. Figure S2

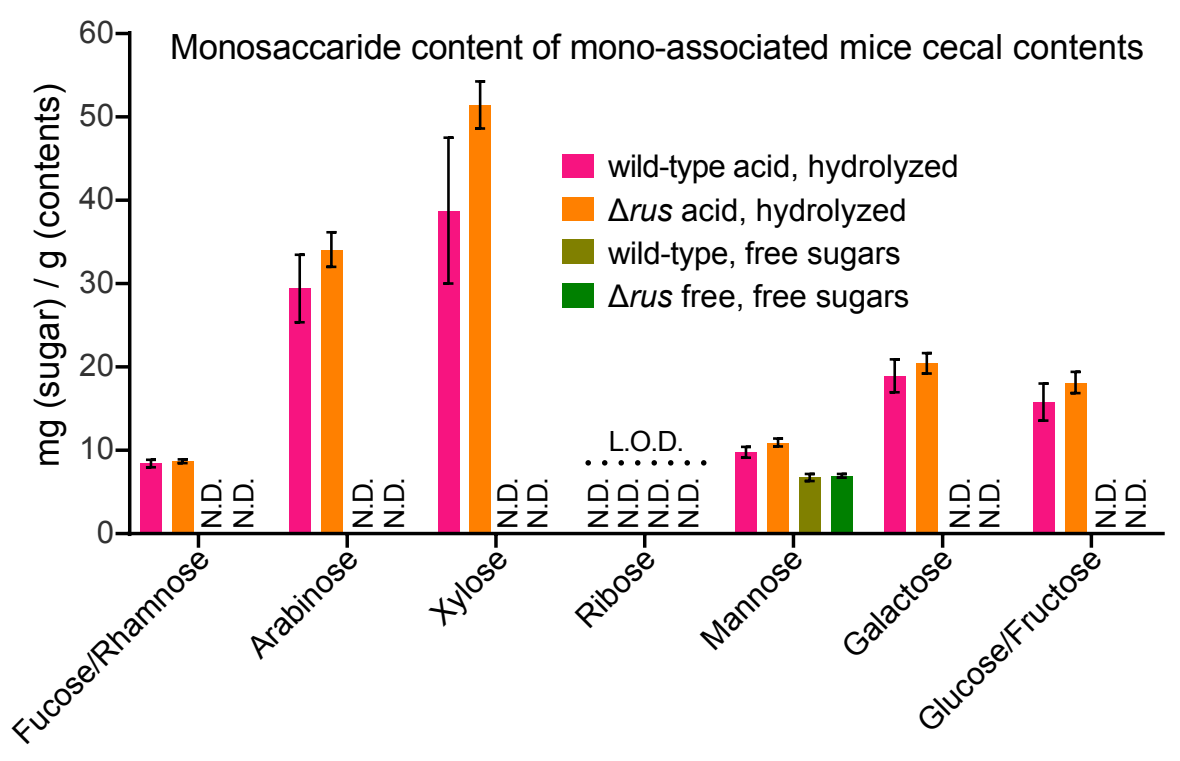


A.

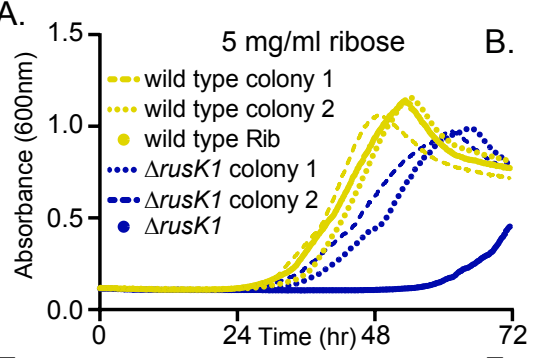

E. 1.5.

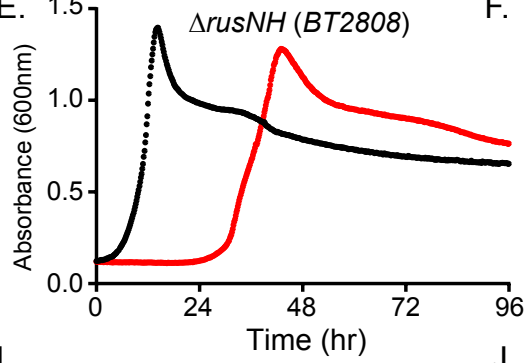

I. 1000 Time (hr)
rusC (BT2805) expression
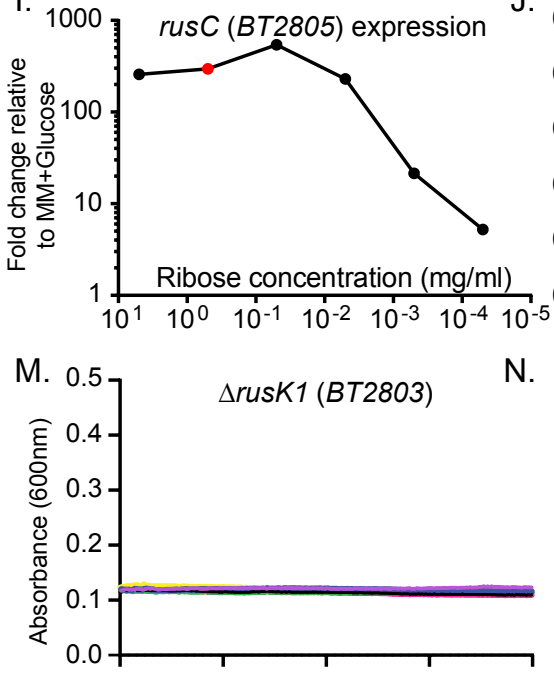

Q. $\left.{ }^{0.5}\right]$ wild-type growth on enzymes $R$.

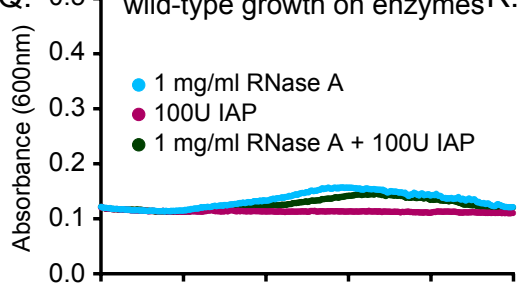

U. 0.57 V.

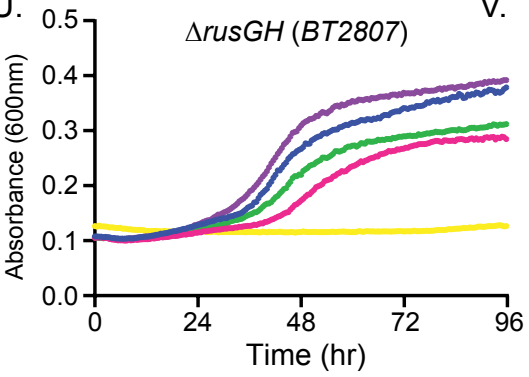

B. 1 $1^{\operatorname{rrusC}(B T 2805)}$
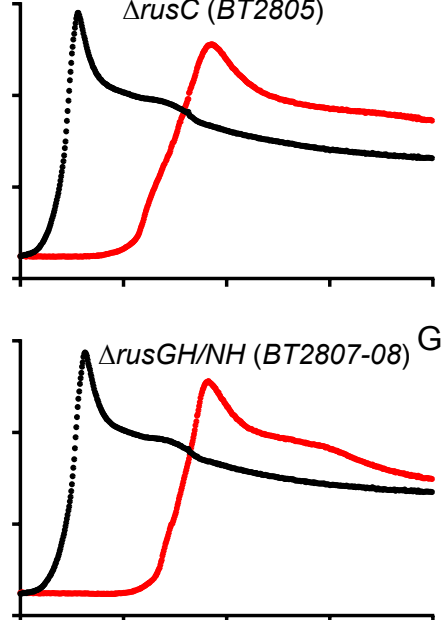

C.

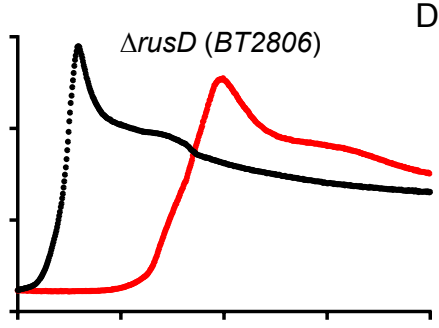

0.5 wild-type growth on

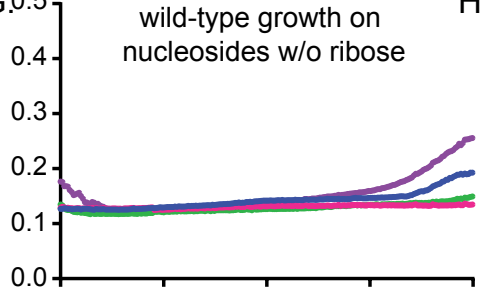

$\mathrm{K}$.
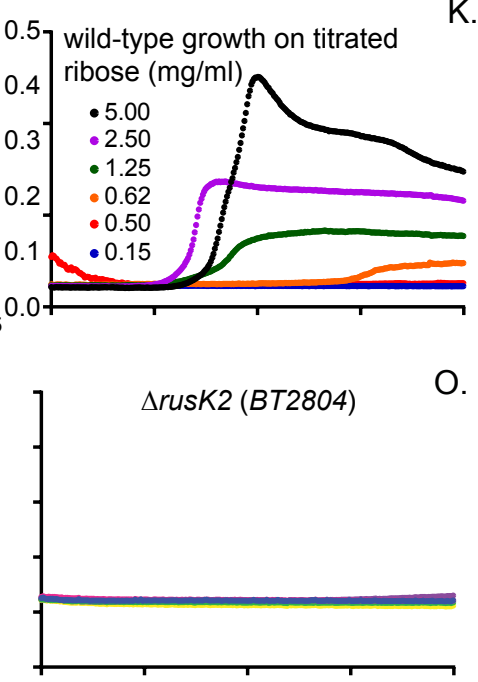

O.

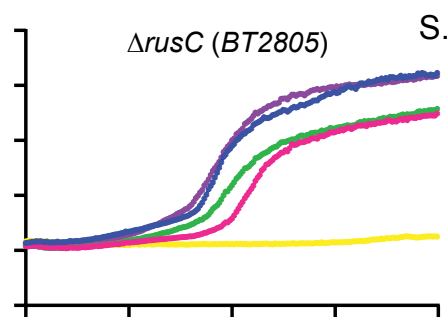

S.

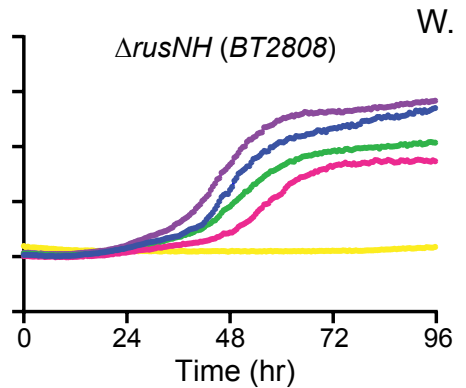

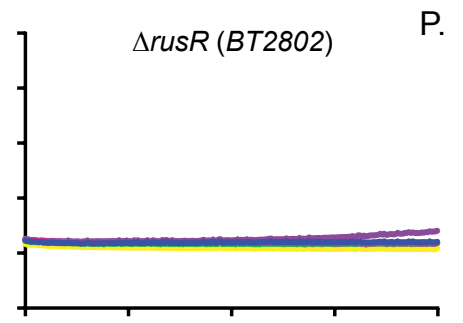
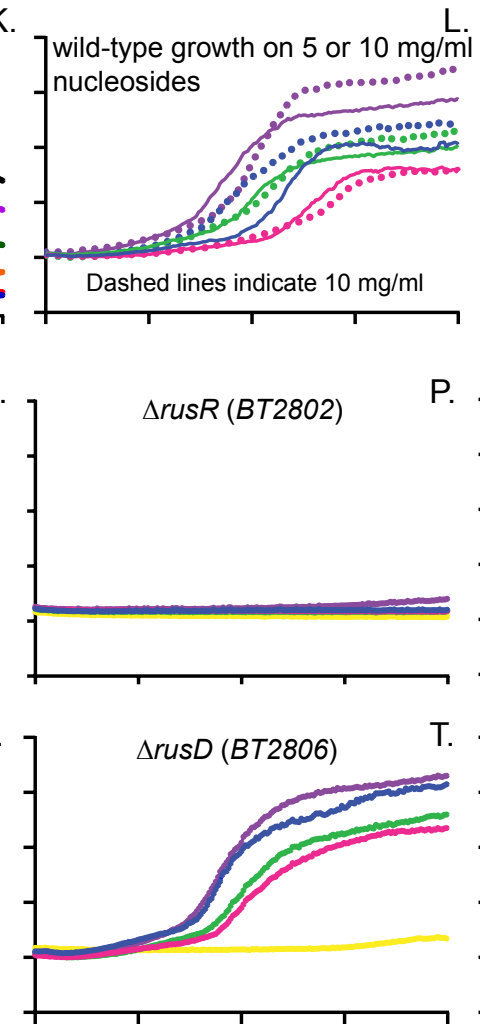

ArusGH/NH (BT2807-08)

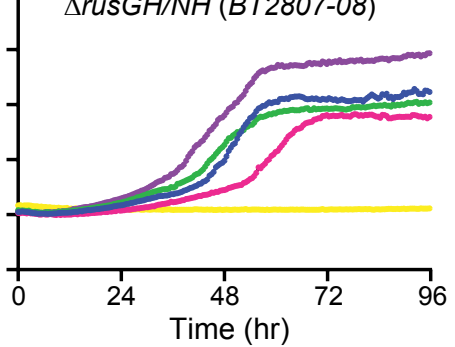

$\mathrm{L}$.
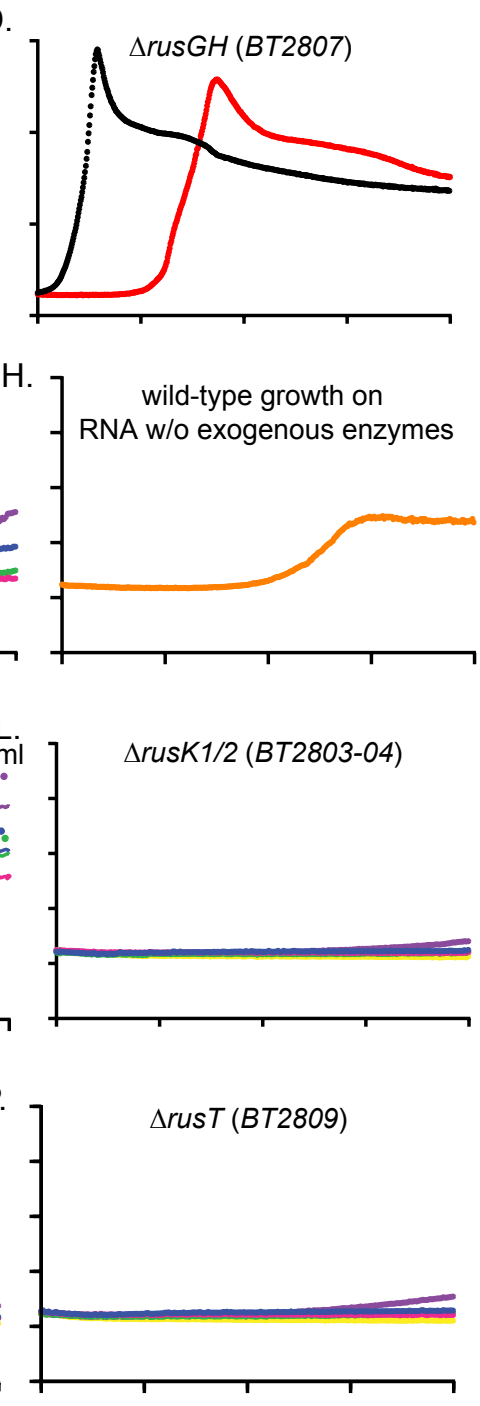

H. wild-type growth on RNA w/o exogenous enzymes

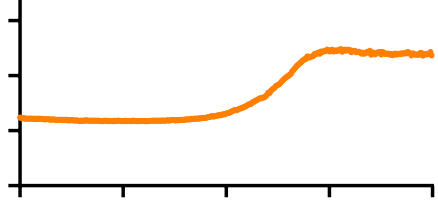

T.

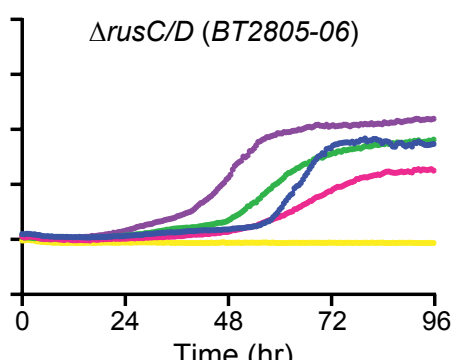

Time (hr)

Glucose $5 \mathrm{mg} / \mathrm{ml}$

Ribose $5 \mathrm{mg} / \mathrm{ml}$

Uridine $5 \mathrm{mg} / \mathrm{ml}$

Cytidine $5 \mathrm{mg} / \mathrm{ml}$

5-Me Uridine $5 \mathrm{mg} / \mathrm{ml}$

Thymidine $5 \mathrm{mg} / \mathrm{ml}$

Ribose $0.5 \mathrm{mg} / \mathrm{ml}$ 
A.

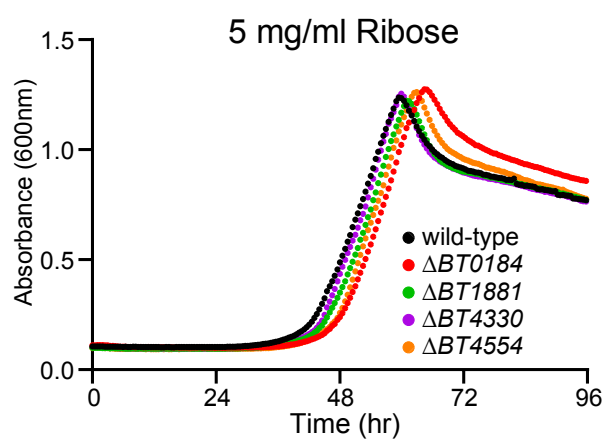

D.

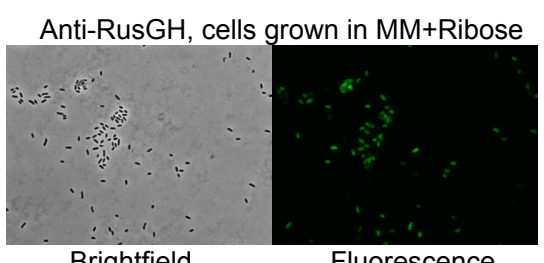

G.

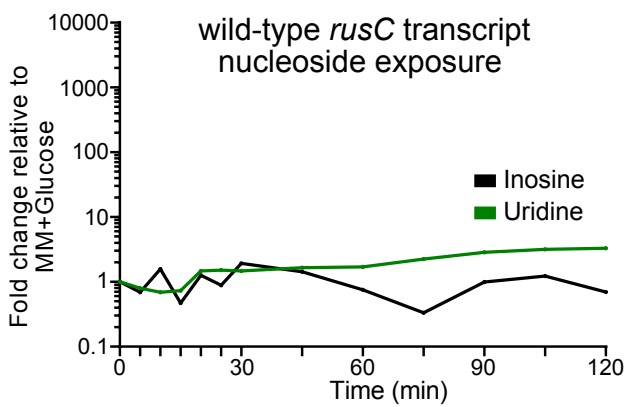

J.

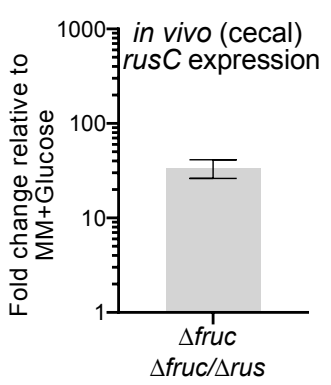

B.

E.

H.
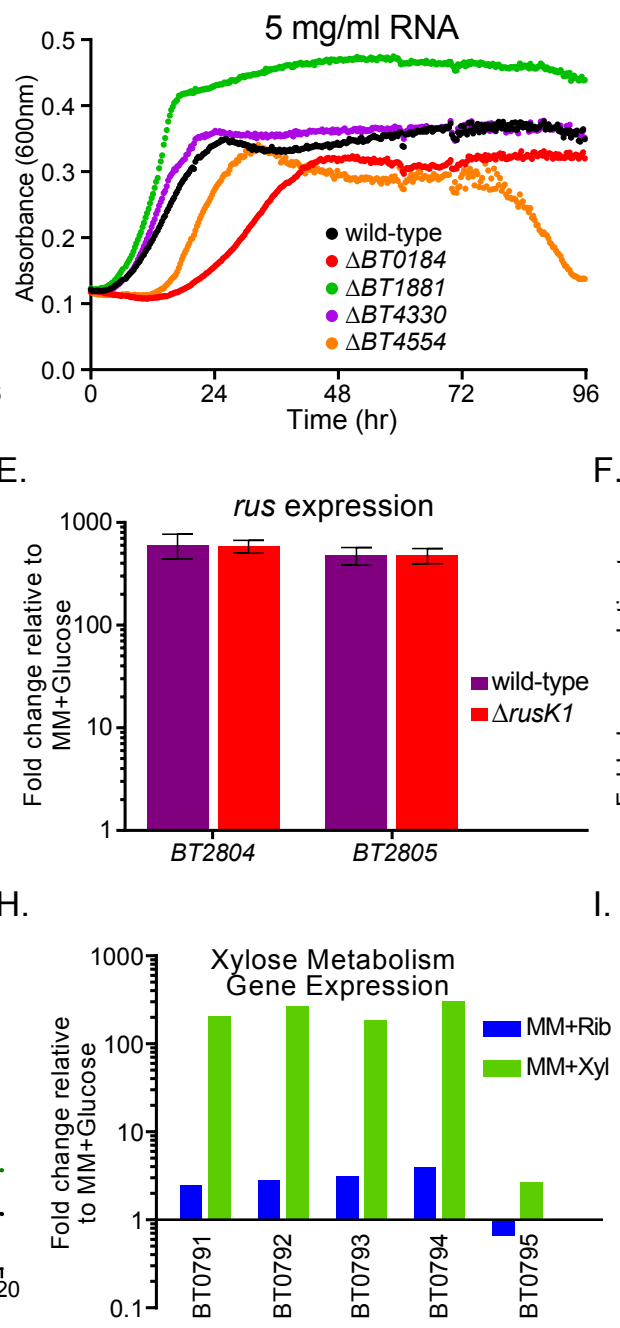

I.

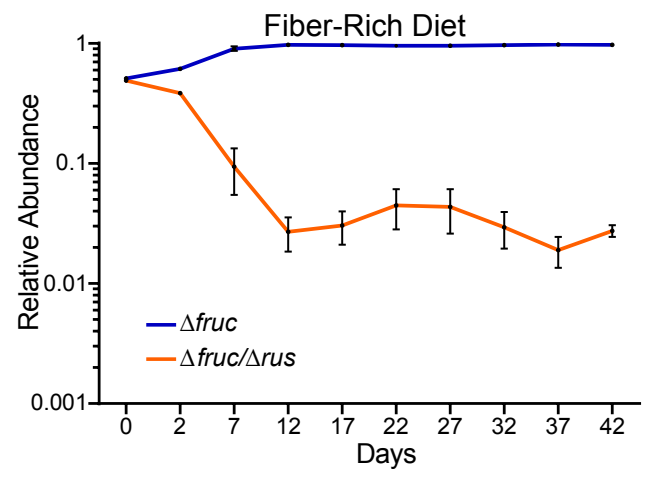

$\mathrm{F}$.

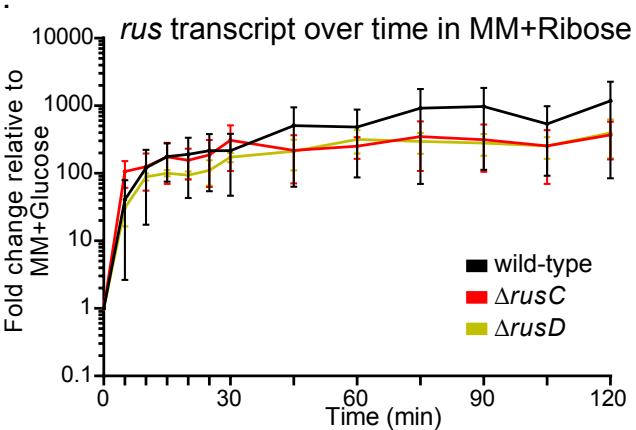

10000 rus transcript over time in MM+Ribose
Sequence alignment of nucleoside

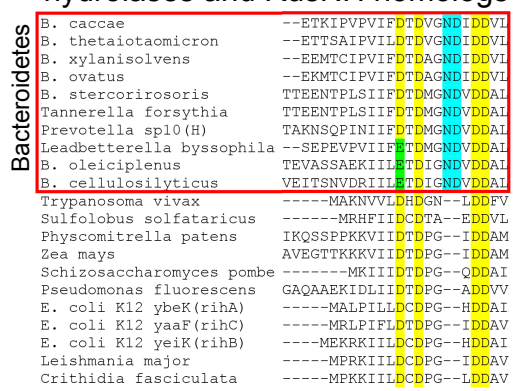

C.

.




\section{Glowacki et al. Figure S5}

Leadbetterella byssophila (1) Type18

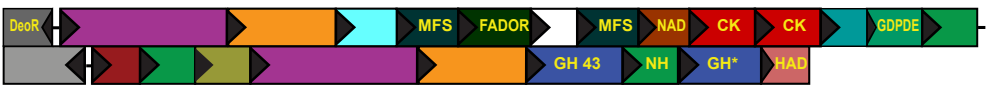

Hymenobacter sp. PAMC 26628 (1) Type 64

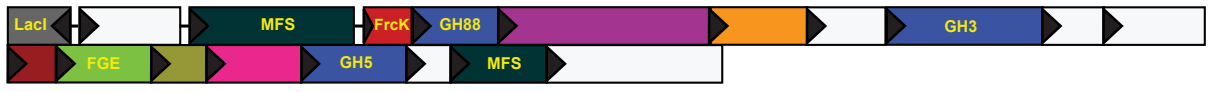

Parapedobacter koreensis Jip14 (1) Type 28

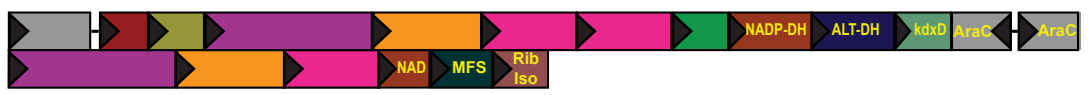

Petrimonas mucosa ING2-E5A (1) Type 52 Alistipes obesi ph8 (1) Type 29

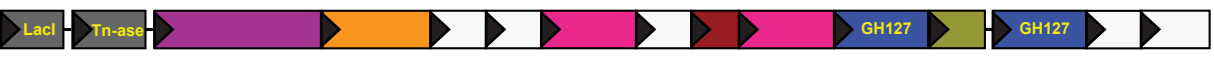

Niabella ginsenosidivorans BS26 (1) Type 23 Sphingobacteriaceae bacterium DW12 (1) Type 57

Pedobacter nyackensis DSM 19625 (1) Type 49 Bacteroidetes-49 (2) Type 60

Porphyromonadaceae bacterium KH3CP3RA (2) Type 22 Prevotella sp. 10(H) (1) Type 15 Porphyromonadaceae bacterium KHP3R9 (1) Type 19

Parapedobacter composti DSM 22900 (1) Type 54 Spirosome linguale DSM 74 (1) Type 27 Bacteroides sp. 14(A) (1) Type 31 P. dentalis (2) / Hallela sergens (1) Type 16 P. multisaccharivorax (1) Type 17 Marinibilia anaerophila DSM 26910 (1) Type 62 Sphingobacterium yanglingense DSM 28353 (1) Type 45 Niabella aurantiaca DSM 17617 (1) Type 47 Pedobacter sp. OK626 (3) Type 50 Siccationidurans arizonensis DSM 17870 (1) Type 56 Sphingobacterium psychroaquaticum DSM 22418 (1) Type 26 Bacteroides sp. 3_1_19 (2) Type 58 Hymenobacter terrenus MIMtkLc17 (1) Type 66 Arenibacter latericius DSM 15913 (1) Type 35 Larkinella arboricola DSM 21851 (1) Type 43 Chitinophagaceae bacterium PMP191F (1) Type 40 Porphyromonadaceae bacterium KH3R12 (1) Type 25 Alistipes indistictus YIT 12060 (1) Type 34 Bacteroidetes bacterium RBG 13_43_22 (2) Type 59

Cytophagales bacterium TFI 002 (1) Type 63 Chitinophagaceae arvensicola DSM 3695 (1) Type 41 Sphingobacterium multivorum DSM 11691 (2) Type 65 Pedobacter heparinus HIM 762-3 DSM 2366 (2) Type 51 Prevotella fusca W1435 (1) Type 20 Cecembia rubra DSM 28057 (1) Type 67 Alkaliflexus imshenetskii Z-7010, DSM 15055 (1) Type 69

Segetibacter koreensis DSM 18137 (1) Type 55 Alistipes ihumii AP11 (1) Type 68

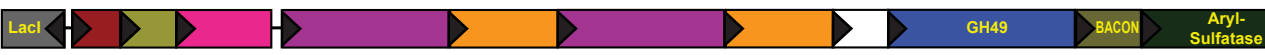

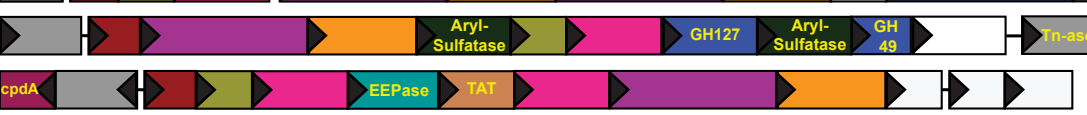

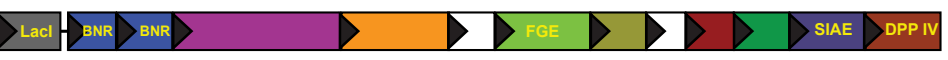
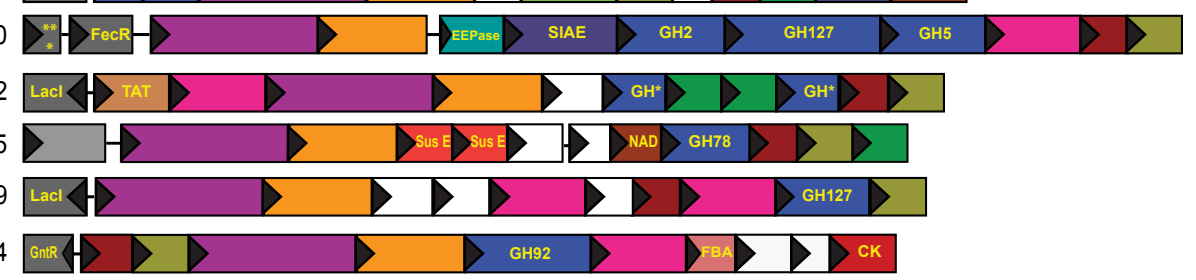

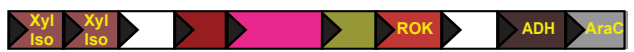
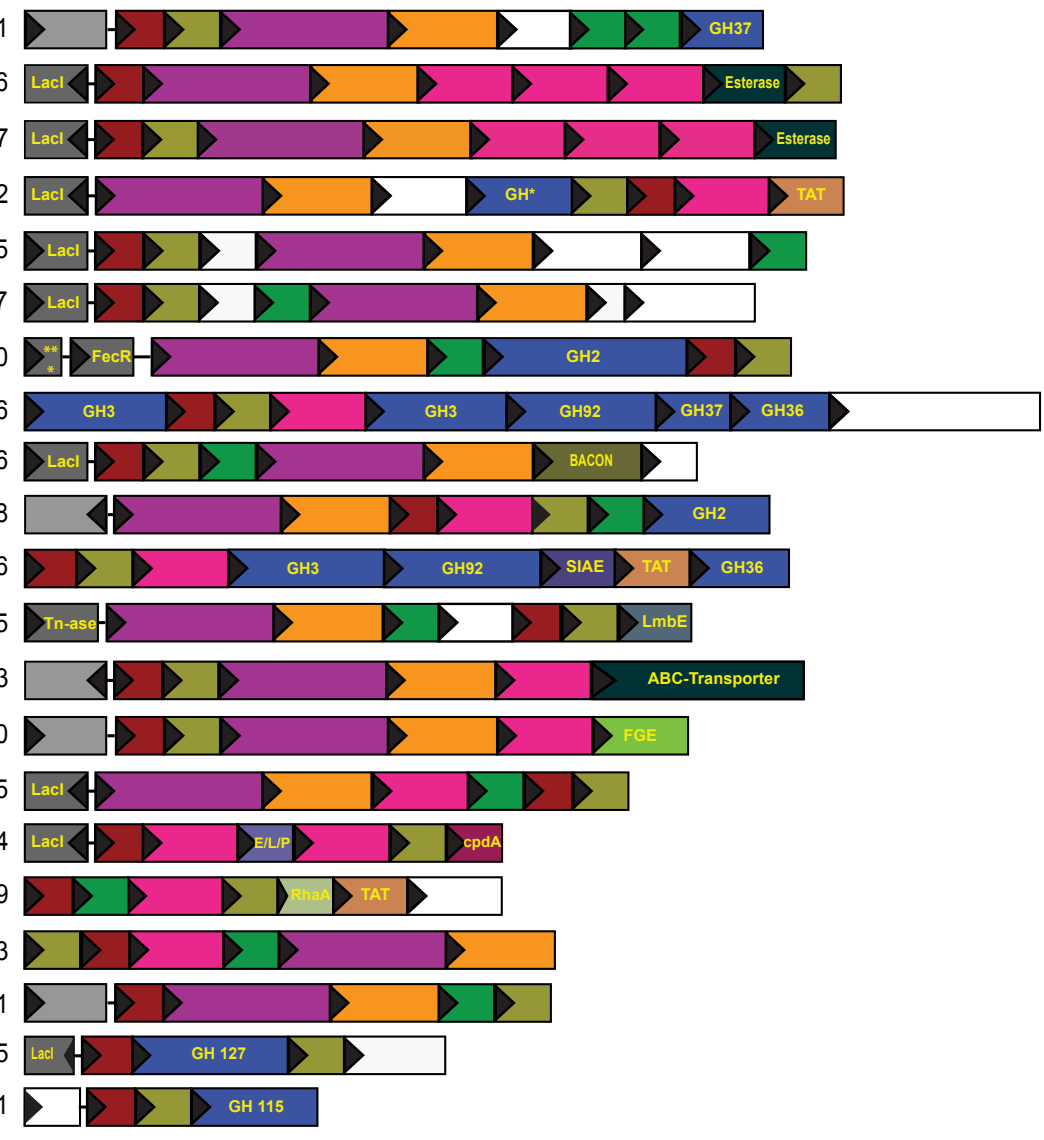

Laci $\langle->>1 /$ Nucleotidas

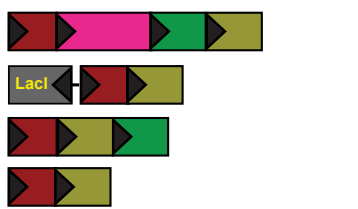

